\title{
Solving all 4-point correlation functions for bosonic open string theory in the high-energy limit
}

\author{
Chuan-Tsung Chan ${ }^{\mathrm{a}}$, Pei-Ming Ho ${ }^{\mathrm{b}}$, Jen-Chi Lee ${ }^{\mathrm{c}}$, \\ Shunsuke Teraguchi ${ }^{\mathrm{d}}$, Yi Yang ${ }^{\mathrm{c}}$
}

a Physics Division, National Center for Theoretical Sciences, Hsinchu, Taiwan, ROC

${ }^{\mathrm{b}}$ Department of Physics, National Taiwan University, Taipei, Taiwan, ROC

c Department of Electrophysics, National Chiao-Tung University, Hsinchu, Taiwan, ROC

d Physics Division, National Center for Theoretical Sciences, Taipei, Taiwan, ROC

Received 25 April 2005; received in revised form 6 July 2005; accepted 15 July 2005

Available online 8 August 2005

\begin{abstract}
We study the implication of decoupling zero-norm states in the high-energy limit, for the 26dimensional bosonic open string theory. Infinitely many linear relations among 4-point functions are derived algebraically, and their unique solution is found. Equivalent results are also obtained by taking the high-energy limit of Virasoro constraints, and as an independent check, we compute all 4-point functions of 3 tachyons and an arbitrary massive state by saddle-point approximation.

(c) 2005 Elsevier B.V. All rights reserved.
\end{abstract}

\section{Introduction}

\subsection{Motivation}

The high-energy limit of string theory is an old subject [1-3]. Its motivation is to find the hypothetical hidden symmetry. It is believed that the higher-spin gauge fields in string

E-mail addresses: ctchan@phys.cts.nthu.edu.tw (C.-T. Chan),pmho@ntu.edu.tw (P.-M. Ho), jcclee@cc.nctu.edu.tw (J.-C. Lee), teraguch@phys.ntu.edu.tw (S. Teraguchi), yiyang@mail.nctu.edu.tw (Y. Yang). 
theory receive their masses via a spontaneous symmetry breaking mechanism $[2,4]$. It is then natural to expect that the gauge fields become effectively massless and the symmetry is restored in the high-energy limit. The symmetry might also explain mysterious correspondences such as dualities and holography.

This subject has been attacked from various directions. One can take the high-energy limit of the worldsheet theory and use it to define string theory in the high-energy limit [5]. However there is a lot of ambiguity in taking the high-energy limit of a worldsheet. It is not clear which choice of the high-energy worldsheet action is the one suitable for the purpose of studying symmetries.

Another approach is to study consistent higher spin gauge theories [6]. It turns out that there is no consistent scale-free nontrivial interactions in the flat spacetime. Usually people study these theories in the AdS background. In general we need a characteristic length scale to define consistent interactions. The string scale $\alpha^{\prime}$ cannot be used because by definition $\alpha^{\prime} \rightarrow \infty$ in the high-energy limit.

The third approach is to examine correlation functions in string theory and look for meaningful patterns in the high-energy limit [1-3]. This is the approach that we will take in this work. We consider the bosonic open string theory and compare correlation functions which are different from each other by a single vertex at the same mass level. We claim that their relative ratios are completely determined at the leading order for any mass level. This should be viewed as the strongest signal there ever was for a symmetry in the high-energy limit.

The main idea of our approach is that the decoupling of zero-norm states strongly restricts the dynamics of string theory [7]. One can show [8,9] that off-shell gauge transformations of Witten's string field theory, after imposing the no-ghost condition, are identical to the on-shell stringy gauge symmetries generated by two types of zero-norm states. In view of the dictating role of gauge symmetry in Witten's SFT, we believe that the existence of the huge gauge symmetry represented by zero-norm states $[10,11]$ fixes the theory uniquely. In particular, we will show in this paper that by taking a self-consistent highenergy limit [12-14] of arbitrary four-point functions, it will allow us to express them at the leading order in terms of those of tachyons. We will also take the $2 \mathrm{D}$ string as an example to establish the connection between the high-energy limit of zero-norm states and the hidden symmetry. In 2D string, we will show that the high-energy limit of $w_{\infty}$ algebra generated by $2 \mathrm{D}$ zero-norm states [15] can be identified with the $w_{\infty}$ symmetry of 2D string [16].

\subsection{Review}

We will focus on 4-point functions in this work, although our discussion can be generalized to higher point correlation functions. Due to Poincaré symmetry, a 4-point function is a function of merely two parameters. Viewing a 4-point function as the scattering amplitude of a two-body scattering process, one can choose the two parameters to be $E$ (one half of the center of mass energy for the incoming particles, i.e., particles 1 and 2 in Fig. 1 , and $\phi$ (the scattering angle between particles 1 and 3). For convenience we will take the center of mass frame and put the momenta of particles 1 and 2 along the $X^{1}$-direction, with 


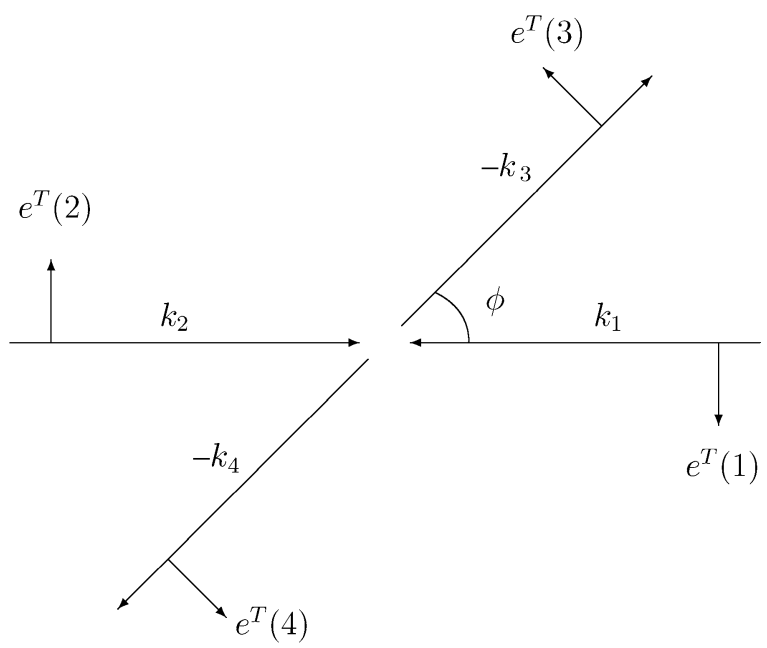

Fig. 1. Kinematic variables in the center of mass frame.

the momenta of particles 3 and 4 on the $X^{1}-X^{2}$ plane. Readers can refer to Appendix A for expressions of the kinematic variables in this frame.

The high-energy limit under consideration is

$$
\alpha^{\prime} E^{2} \rightarrow \infty, \quad \phi=\text { fixed }
$$

Based on the saddle-point approximation of Gross and Mende [1], Gross and Manes [3] computed the high-energy limit of 4-point functions in the bosonic open string theory. To explain their result, let us first define our notations and conventions. For a particle of momentum $k$, we define an orthonormal basis of polarizations $\left\{e^{P}, e^{L}, e^{T_{i}}\right\}$. The momentum polarization $e^{P}$ is proportional to $k$, the longitudinal polarization $e^{L}$ is the space-like unit vector whose spatial component is proportional to that of $k$, and $e^{T_{i}}$ are the space-like unit-vectors transverse to the spatial momentum. As an example, for $k$ pointing along the $X^{1}$-direction,

$$
k=\left(k^{0}, k^{1}, k^{2}, \ldots, k^{25}\right)=(E, p, 0, \ldots, 0), \quad p>0,
$$

the basis of polarization is

$$
\begin{aligned}
e^{P} & =\frac{1}{m}\left(\sqrt{p^{2}+m^{2}}, p, 0,0, \ldots, 0\right), \quad e^{L}=\frac{1}{m}\left(p, \sqrt{p^{2}+m^{2}}, 0,0, \ldots, 0\right), \\
e^{T_{i}} & =(0,0, \ldots, 1, \ldots),
\end{aligned}
$$

where $m$ is the mass of the particle. In general, $e^{T_{i}}$ (for $i=3, \ldots, 25$ ) is just the unit vector in the $X^{i}$-direction, and the definitions of $e^{P}, e^{L}$ and $e^{T_{2}}$ will depend on the motion of the particle. For $e^{T_{2}}$, which is parallel to the scattering plane, we denote it by $e^{T}$ (see Fig. 1 and Appendix A). The orientations of $e^{T_{2}}$ for each particle are fixed by the right-hand rule, $\vec{k} \times e^{T_{2}}=e^{T_{3}}$, where $\vec{k}$ is the spatial momentum of 4-vector $k$. We will use the notation $\partial^{n} X^{A} \equiv e^{A} \cdot \partial^{n} X$ for $A=P, L, T, T_{i}$. 
Each vertex is a polynomial of $\left\{\partial^{n} X^{A}\right\}$ times the exponential factor $\exp (i k \cdot X)$. Among all possible choices of polarizations for the 4 vertices in a 4-point function, we now argue that only the polarizations $L$ and $T$ need to be considered. The polarization $P$ can be gauged away using zero-norm states [9]. To see why we can ignore all $T_{i}$ 's except $T$, we note that a prefactor $\partial^{n} X^{A}$ can be contracted with the exponent $i k \cdot X$ of another vertex. The contribution of this contraction to a scattering amplitude is proportional to $k^{A} \sim E$. If $k^{A} \neq 0$ (i.e., if $A=L$ or $T$ ), this is much more important in the high-energy limit than a contraction with another prefactor $\partial^{m} X^{B}$, which gives $\eta^{A B} \sim E^{0}$. Therefore, if all polarizations in all vertices are chosen to be either $L$ or $T$, the resulting 4-point function will dominate over other choices of polarizations.

According to the zeroth-order saddle-point approximation [1], the leading order of a 4-point function is

$$
\left\langle V\left(k_{1}\right) V\left(k_{2}\right) V\left(k_{3}\right) V\left(k_{4}\right)\right\rangle \sim\left(\prod_{a=1}^{4} v_{a}\right) \mathcal{T} .
$$

Here $\mathcal{T}$ is the correlation function of 4 tachyons, according to the following rules

$$
\begin{aligned}
& \frac{1}{(n-1) !} \partial^{n} X^{T} \rightarrow i \frac{E \sin \phi}{(-\lambda)^{n}}, \\
& \frac{1}{(n-1) !} \partial^{n} X^{L} \rightarrow-i \frac{\lambda^{n-1}-1}{\lambda-1} \frac{E^{2} \sin ^{2} \phi}{2 M(-\lambda)^{n}},
\end{aligned}
$$

where $^{1}$

$$
\lambda=\sin ^{2} \frac{\phi}{2} .
$$

A peculiar feature of (6) is that it vanishes for $n=1$

$$
\partial X^{L} \rightarrow 0 \text {. }
$$

Does this mean that all 4-point functions involving $\partial X^{L}$ are subleading? The answer depends on how we define the notion of "subleading". What is the reference 4-point function to be compared with?

Note that it is impossible to have a universal notion of "leading order" for all 4-point functions, since 4-point functions with vertices at higher mass levels typically dominate over those with vertices at lower mass levels in the high-energy limit. Having a well defined limit for all correlation functions is also contradictory to the fact that there is no consistent interacting higher-spin gauge field theory in the flat spacetime.

We propose that the appropriate question to ask is: How will a 4-point function change if we replace a vertex by another vertex at the same mass level? For example, we can replace a factor $\partial X^{L}$ by $\partial X^{T}$ in one of the vertices. Naively, the former with $\partial X^{L}$ should dominate over the latter in the high-energy limit since the components of $e^{L}$ scale as $E^{1}$ and those of $e^{T}$ scale as $E^{0}$. However, Eq. (8) tells us that $\partial X^{L}$ does not live up to this expectation.

1 However, see correction in Ref. [14]. 
Nevertheless, it does not imply that $\partial X^{L}$ is subleading compared with $\partial X^{T}$. It depends on the rest of the terms in the prefactor.

The purpose of our work is to find linear relations among 4-point functions in the highenergy limit as a signature of the hidden symmetry proposed in [1]. This is not manifest in the saddle-point result (5), (6), which holds for the naive leading order (0th order saddlepoint approximation). One of the main reasons is that they ignored vertices involving $\partial X^{L}$. Another main reason is that they did not try to determine the precise power factor of $E$ [14] in a 4-point function (a higher order correction to the saddle-point approximation is needed), which is crucial for examining linear relations among 4-point functions.

The proper notion of "leading order" in the high-energy limit such that linear relations among 4-point functions can be established was first discovered in [12,13]. A purely algebraic approach utilizing zero-norm states was developed there to derive the linear relations explicitly for the first few mass levels. This approach was further explored to obtain stronger results, and its connection/difference from the saddle-point computation was explained in detail in [14].

The central idea behind the algebraic approach used in [12-14] was the decoupling of zero-norm states (i.e., the requirement of gauge invariance). A crucial step in the derivation is to replace the polarization $e^{P}$ by $e^{L}$ in the zero-norm states. It is assumed that, while zero-norm states decouple at all energies, the replacement leads to states that are decoupled at high energies.

The new achievements of this paper include:

(1) We studied the implication of the decoupling of zero-norm states in the high-energy limit. The 4-point functions at the leading order are specified for all mass levels.

(2) Infinite linear relations among 4-point functions are obtained for all mass levels. The ratio of any two 4-point functions at the leading order is uniquely determined.

(3) We developed a "dual" description of the algebraic approach using the spurious states, which is to take high-energy limit of the Virasoro constraints.

(4) Using saddle-point approximation, we explicitly computed all 4-point functions for 3 tachyons and one arbitrary massive state, and verified the linear relations obtained via algebraic methods.

\section{Main results}

For brevity, we will refer to all 4-point functions different from each other by a single vertex at the same mass level as a "family". When we compare members of a family, we only need to specify the vertex which is changed.

A 4-point function will be said to be at the leading order if it is not subleading to any of its siblings. We will ignore those that are not at the leading order. Our aim is to find the numerical ratios of all 4-point functions in the same family at the leading order. Apparently, there are more 4-point functions at the leading order at higher mass levels. Our goal may seem insurmountable at first sight.

Saving the derivation for later, we give our main results here. A 4-point function is at the leading order if and only if the vertex $V$ under comparison is a linear combination of 
vertices of the form

$$
V^{(n, m, q)}(k)=\left(\partial X^{T}\right)^{n-m-2 q}\left(\partial X^{L}\right)^{m}\left(\partial^{2} X^{L}\right)^{q} e^{i k \cdot X},
$$

where

$$
n \geqslant m+2 q, \quad m, q \geqslant 0 .
$$

The corresponding states are of the form

$$
\left(\alpha_{-1}^{T}\right)^{n-m-2 q}\left(\alpha_{-1}^{L}\right)^{m}\left(\alpha_{-2}^{L}\right)^{q}|0, k\rangle .
$$

The mass squared is $2(n-1)$. All other states involving $\alpha_{-2}^{T}, \alpha_{-3}^{A}, \ldots$ are subleading.

Using the notation ${ }^{2}$

$$
\mathcal{T}^{(n, m, q)}=\left\langle V_{1} V^{(n, m, q)}(k) V_{3} V_{4}\right\rangle,
$$

all linear relations among different choices of $V^{(m, n, q)}$ (obtained from the decoupling of spurious states at high energies) can be solved by the simple expression

$$
\begin{aligned}
& \lim _{E \rightarrow \infty} \frac{\mathcal{T}^{(n, 2 m, q)}}{\mathcal{T}^{(n, 0,0)}}=\left(-\frac{1}{\hat{m}}\right)^{2 m+q}\left(\frac{1}{2}\right)^{m+q}(2 m-1) ! ! \\
& \lim _{E \rightarrow \infty} \frac{\mathcal{T}^{(n, 2 m+1, q)}}{\mathcal{T}^{(n, 0,0)}}=0,
\end{aligned}
$$

where $\hat{m}=\sqrt{2(n-1)}$. This formula tells us how to trade $\partial X^{L}$ and $\partial^{2} X^{L}$ for $\partial X^{T}$, so that all 4-point functions can be related to the one involving only $\partial X^{T}$ in $V_{2}$. The formula above applies equally well to all vertices.

Since we know the value of a representative 4-point function [12,14]

$$
\mathcal{T}_{n_{1} n_{2} n_{3} n_{4}}^{T^{1} . . T^{2} \cdot . T^{4} \cdot .}=(-1)^{n_{2}+n_{4}}\left[2 E^{3} \sin \phi_{\mathrm{CM}}\right]^{\Sigma n_{i}} \mathcal{T}\left(\Sigma n_{i}\right),
$$

where

$$
\begin{aligned}
\mathcal{T}(n)= & \sqrt{\pi}(-1)^{n-1} 2^{-n} E^{-1-2 n}\left(\sin \frac{\phi_{\mathrm{CM}}}{2}\right)^{-3}\left(\cos \frac{\phi_{\mathrm{CM}}}{2}\right)^{5-2 n} \\
& \times \exp \left(-\frac{s \ln s+t \ln t-(s+t) \ln (s+t)}{2}\right)
\end{aligned}
$$

is the high-energy limit of $\frac{\Gamma\left(-\frac{s}{2}-1\right) \Gamma\left(-\frac{t}{2}-1\right)}{\Gamma\left(\frac{u}{2}+2\right)}$ with $s+t+u=2 \Sigma n_{i}-8$, and we have calculated it up to the next leading order in $E$. In Eq. (15), $n_{i}$ is the number of $T^{i}$ of the $i$ th vertex operators and $T^{i}$ is the transverse direction of the $i$ th particle. We can immediately write down the explicit expression of a 4-point function if all vertices are nontrivial at the leading order.

\footnotetext{
2 More rigorously, $V_{2}$ needs to be a physical state in order for the correlation function to be well-defined. We should keep in mind that our results should be applied to suitable linear combinations of (9), possibly together with subleading states, to satisfy Virasoro constraints.
} 


\section{Linear relations among 4-point functions}

Before we go on, we recall some terminology used in the old covariant quantization. A state $|\psi\rangle$ in the Hilbert space is physical if it satisfies the Virasoro constraints

$$
\left(L_{n}-\delta_{n}^{0}\right)|\psi\rangle=0, \quad n \geqslant 0 .
$$

Since $L_{n}^{\dagger}=L_{-n}$, states of the form

$$
L_{-n}|\chi\rangle
$$

are orthogonal to all physical states, and they are called spurious states. Zero-norm states are spurious states that are also physical. They correspond to gauge symmetries. In the old covariant first quantization spectrum of open bosonic string theory, the solutions of physical state conditions include positive-norm propagating states and two types of zeronorm states. The latter are [7]

Type I: $\quad L_{-1}|x\rangle, \quad$ where $L_{1}|x\rangle=L_{2}|x\rangle=0, L_{0}|x\rangle=0$,

$$
\text { Type II: } \quad\left(L_{-2}+\frac{3}{2} L_{-1}^{2}\right)|\tilde{x}\rangle, \quad \text { where } L_{1}|\tilde{x}\rangle=L_{2}|\tilde{x}\rangle=0,\left(L_{0}+1\right)|\tilde{x}\rangle=0 .
$$

In this section we derive the linear relations among all amplitudes in the same family by taking the high-energy limit of zero-norm states (HZNS). Solutions of HZNS for some low lying mass level are presented in Appendix B. The first step in the derivation is to identify the class of states that are relevant, i.e., those at the leading order. As we explained in Section 1.2, we only need to consider the polarizations $e^{T}$ and $e^{L}$.

To get a rough idea about how each vertex operator scales with $E$ in the high-energy limit, we associate a naive dimension to each prefactor $\partial^{m} X^{A}$ according to the following rule

$$
\partial^{m} X^{T} \rightarrow 1, \quad \partial^{m} X^{L} \rightarrow 2 .
$$

The reason is the following. Each factor of $\partial^{m} X^{\mu}$ has the possibility of contracting with the exponent $i k_{i} \cdot X$ of another vertex operator so that it scales like $E$ in the high-energy limit. Furthermore, components of the polarization vectors $e^{T}$ and $e^{L}$ scale with $E$ like $E^{0}$ and $E^{1}$, respectively.

When we compare vertex operators at the same mass level, the sum of all the integers $m$ in $\partial^{m} X^{A}$ is fixed. Roughly speaking, it is advantageous to have many $\partial X^{A}$ than having fewer number of $\partial^{m} X^{A}$ with $m>1$. For example, at the first massive level, the vertex operator $\partial X^{T} \partial X^{T} e^{i k \cdot X}$ has a larger naive dimension than $\partial^{2} X^{T} e^{i k \cdot X}$.

The counting of the naive dimension does not take into consideration the possibility that the coefficient of the leading order term happens to vanish by cancellation. The true dimension of a vertex operator can be lower than its naive dimension, although the reverse never happens.

Through experiences accumulated from explicit computations [12-14], we find that the highest spin vertex

$$
\left(\partial X^{T}\right)^{n} e^{i k \cdot X} \leftrightarrow\left(\alpha_{-1}^{T}\right)^{n}|0, k\rangle
$$


is always at the leading order in its family. Since the naive dimension of this state equals its true dimension, any state with a lower naive dimension than this vertex operator can be ignored. This implies that we can immediately throw away a lot of vertex operators at each mass level, but there are still many left. The problem is that, although there are disadvantages to have $\partial^{m} X^{T}$ with $m \geqslant 2$ or $\partial^{m} X^{L}$ with $m \geqslant 3$ compared with having $\left(\partial X^{T}\right)^{m}$, it may be possible that having extra factors of $\partial X^{L}$, which has a higher naive dimension than $\partial X^{T}$, can compensate the disadvantage of these factors. However, explicit computations at the first few massive levels showed that this never happens.

We will now argue why this is generically true, and show in this subsection that the only states that will survive the high-energy limit at level $n$ are of the form

$$
|n, 2 m, q\rangle \equiv\left(\alpha_{-1}^{T}\right)^{n-2 m-2 q}\left(\alpha_{-1}^{L}\right)^{2 m}\left(\alpha_{-2}^{L}\right)^{q}|0 ; k\rangle .
$$

Our argument is essentially based on the decoupling of zero-norm states in the highenergy limit. However, note that when we take the high-energy limit, that is, when we replace $e^{P}$ by $e^{L}$ and ignore subleading terms in the $1 / E^{2}$ expansion, ${ }^{3}$ the zero-norm states become positive norm states, although we will still call them "high-energy zero-norm states", or HZNS for short. (This is why it is possible to derive relations among positivenorm physical states by taking the high-energy limit of Ward identities. See Appendix B, for examples.) As such, it is not essential to maintain the zero-norm condition, and we can simply take the high-energy limit of spurious states. It can be shown that, as far as the final results are concerned, the decoupling of those spurious states we are going to use in this paper are equivalent to the decoupling of high-energy zero-norm states (HZNS).

Thanks to the Virasoro algebra, we only need two Virasoro operators

$$
\begin{aligned}
& L_{-1}=\frac{1}{2} \sum_{n \in \mathbb{Z}} \alpha_{-1+n} \cdot \alpha_{-n}=\hat{m} \alpha_{-1}^{P}+\alpha_{-2} \cdot \alpha_{1}+\cdots, \\
& L_{-2}=\frac{1}{2} \sum_{n \in \mathbb{Z}} \alpha_{-2+n} \cdot \alpha_{-n}=\frac{1}{2} \alpha_{-1} \cdot \alpha_{-1}+\hat{m} \alpha_{-2}^{P}+\alpha_{-3} \cdot \alpha_{1}+\cdots
\end{aligned}
$$

to generate all spurious states. Here $\hat{m}$ is the mass operator, i.e., $\hat{m}^{2}=-k^{2}$ when acting on the state $|0, k\rangle$.

\subsection{Irrelevance of other states}

To prove that only states of the form (23) are at the leading order, we shall prove that (i) any state which has an odd number of $\alpha_{-1}^{L}$ is irrelevant (i.e., subleading in the highenergy limit), and (ii) any state involving a creation operator whose naive dimension is less than its mode index $n$, i.e., states belonging to

$$
\left\{\alpha_{-n}^{L}, n>2 ; \alpha_{-m}^{T}, m>1\right\}
$$

is also irrelevant. We proceed by mathematical induction.

\footnotetext{
3 Strictly speaking, we need to justify the replacement $e^{P} \rightarrow e^{L}$. This is not totally trivial, and will be treated with more rigor in a forthcoming paper.
} 
First we prove that any state which has a single factor of $\alpha_{-1}^{L}$ is irrelevant, and that any state with two $\alpha_{-1}^{L}$ 's is irrelevant if it contains an operator of naive dimension less than its index.

Consider the HZNS $L_{-1} \chi$ where $\chi$ is any state without any $\alpha_{-1}^{L}$, and it is at level $(n-1)$. Note that, except $\alpha_{-1}^{L}$, the naive dimension of an operator is always less than or equal to its index (we exclude $\alpha_{-1}^{P}$ as mentioned above). This means that the naive dimension of $\chi$ is less than or equal to $(n-1)$. Since we know that at level $n$, the state Eq. (23) has true dimension $n$, when computing $L_{-1} \chi$ in the high-energy limit, we can ignore everything with naive dimension less than $n$. This means that we need $L_{-1}$ to increase the naive dimension of $\chi$ by no less than 1 . In the high-energy limit of $L_{-1}$

$$
L_{-1} \rightarrow \hat{m} \alpha_{-1}^{L}+\alpha_{-2}^{L} \alpha_{1}^{L}+\alpha_{-2}^{T} \alpha_{1}^{T}+\cdots,
$$

only the first term will increase the naive dimension of $\chi$ by 1 . All the rest do not change the naive dimension. This means that, to the leading order,

$$
L_{-1 \chi} \sim \hat{m} \alpha_{-1}^{L} \chi
$$

This is a state with a single factor of $\alpha_{-1}^{L}$ and it is a HZNS, so it should be decoupled in the high-energy limit.

Now consider an arbitrary state $\chi$ at level $(n-1)$ which has a single factor of $\alpha_{-1}^{L}$. If $\chi$ involves any operator whose naive dimension is less than its index, the naive dimension of $\chi$ is at most $(n-1)$. In the high-energy limit

$$
L_{-1} \chi \rightarrow \hat{m} \alpha_{-1}^{L} \chi+\alpha_{-2}^{L} \alpha_{1}^{L} \chi+\cdots,
$$

except the first two terms, all other terms are irrelevant because they contain a single factor of $\alpha_{-1}^{L}$. As the second term has a naive dimension $(n-1)$ and can be ignored, we conclude that $\alpha_{-1}^{L} \chi$ is irrelevant.

The next step in mathematical induction is to show that if (a) states with $(2 m-1)$ factors of $\alpha_{-1}^{L}$ are irrelevant, and (b) states with $2 m$ factors of $\alpha_{-1}^{L}$ are still irrelevant if it also contains any of the operators in (26), then we can prove that both statements are also valid for $m \rightarrow m+1$.

Suppose $\chi$ is an arbitrary state at level $(n-1)$ which has $2 m$ factors of $\alpha_{-1}^{L}$ 's. The high-energy limit of $L_{-1} \chi$ is given by (29). The second term has $(2 m-1)$ factors of $\alpha_{-1}^{L}$ and is irrelevant. The rest of the terms, except the first, are irrelevant because they contains at least one operator from the set (26). Hence the first term is a HZNS and is irrelevant. We have proved our first claim for $(m+1)$, i.e., a state with $(2 m+1)$ factors of $\alpha_{-1}^{L}$ decouple at high energies.

Similarly, consider the case when $\chi$ is at level $(n-1)$ and has $(2 m-1)$ factors of $\alpha_{-1}^{L}$. Furthermore we assume that it involves operators from the set (26). Then the first term in (29) is what we want to prove to be irrelevant. The second term is irrelevant because we have just proved that a state with $(2 m+1)$ factors of $\alpha_{-1}^{L}$ is irrelevant. The rest of the terms are irrelevant because they have $(2 m-1) \alpha_{-1}^{L}$ 's. Thus we conclude that both claims are correct for $m+1$ as well. The mathematical induction is complete. 


\subsection{Linear relations}

According to the previous subsection, only states of the form (23) are relevant in the high-energy limit. The mass of the state is $\sqrt{2(n-1)}$. The 4-point function associated with $|n, m, q\rangle$ will be denoted $\mathcal{T}^{(n, m, q)}$. The aim of this subsection is to find the ratio between a generic $\mathcal{T}^{(n, m, q)}$ and the reference 4-point function, which is taken to be $\mathcal{T}^{(n, 0,0)}$.

Consider the HZNS

$$
L_{-1}|n-1,2 m-1, q\rangle \simeq \hat{m}|n, 2 m, q\rangle+(2 m-1)|n, 2 m-2, q+1\rangle,
$$

where many terms are omitted because they are not of the form (23). This implies that

$$
\mathcal{T}^{(n, 2 m, q)}=-\frac{2 m-1}{\hat{m}} \mathcal{T}^{(n, 2 m-2, q+1)} .
$$

Using this relation repeatedly, we get

$$
\mathcal{T}^{(n, 2 m, q)}=\frac{(2 m-1) ! !}{(-\hat{m})^{m}} \mathcal{T}^{(n, 0, m+q)},
$$

where the double factorial is defined by $(2 m-1) ! !=\frac{(2 m) !}{2^{m} m !}$.

Next, consider another class of HZNS

$$
L_{-2}|n-2,0, q\rangle \simeq \frac{1}{2}|n, 0, q\rangle+\hat{m}|n, 0, q+1\rangle .
$$

Again, irrelevant terms are omitted here. From this we deduce that

$$
\mathcal{T}^{(n, 0, q+1)}=-\frac{1}{2 \hat{m}} \mathcal{T}^{(n, 0, q)},
$$

which leads to

$$
\mathcal{T}^{(n, 0, q)}=\frac{1}{(-2 \hat{m})^{q}} \mathcal{T}^{(n, 0,0)} .
$$

Our main result (13) is an immediate result of combining (32) and (35).

\section{Linear relations from Virasoro constraints}

In this section we will establish a "dual description" of our approach explained above. The notion dual to the decoupling of high-energy zero-norm states is Virasoro constraints.

Let us briefly explain how to proceed. First write down a state at a given mass level as linear combination of states of the form Eq. (11) with undetermined coefficients, which are interpreted as the Fourier components of spacetime fields. Requiring that the Virasoro generators $L_{1}$ and $L_{2}$ annihilate the state implies several linear relations on the coefficients. The linear relations can then be solved to obtain ratios among all fields.

To compare the results of the two dual descriptions, we note that the correlation functions can be interpreted as source terms for the particle corresponding to a chosen vertex. Thus the ratios among sources should be the same as the ratios among the fields, since all fields of the same mass have the same propagator. However, some care is needed for the normalization of the field variables. One should use BPZ conjugates to determine the norm of a state and normalize the fields accordingly. 


\subsection{Examples}

To illustrate how Virasoro constraints can be used to derive linear relations among scattering amplitudes at high energies, we give some explicit examples in this subsection. We will calculate the proportionality constants among high-energy scattering amplitudes of different string states up to mass levels $m^{2}=8$. The results are of course consistent with those of previous work [12,13] using high-energy zero-norm states.

4.1.1. $m^{2}=4$

The most general form of physical states at mass level $m^{2}=4$ are given by

$$
\left[\epsilon_{\mu \nu \lambda} \alpha_{-1}^{\mu} \alpha_{-1}^{\nu} \alpha_{-1}^{\lambda}+\epsilon_{(\mu \nu)} \alpha_{-1}^{\mu} \alpha_{-2}^{\nu}+\epsilon_{[\mu \nu]} \alpha_{-1}^{\mu} \alpha_{-2}^{\nu}+\epsilon_{\mu} \alpha_{-3}^{\mu}\right]|0, k\rangle .
$$

The Virasoro constraints are

$$
\begin{aligned}
& \epsilon_{(\mu \nu)}+\frac{3}{2} k^{\lambda} \epsilon_{\mu \nu \lambda}=0, \\
& -k^{\nu} \epsilon_{[\mu \nu]}+3 \epsilon_{\mu}-\frac{3}{2} k^{\nu} k^{\lambda} \epsilon_{\mu \nu \lambda}=0, \\
& 2 k^{\nu} \epsilon_{[\mu \nu]}+3 \epsilon_{\mu}-3\left(k^{\nu} k^{\lambda}-\eta^{\nu \lambda}\right) \epsilon_{\mu \nu \lambda}=0 .
\end{aligned}
$$

By replacing $P$ by $L$, and ignoring irrelevant states (we have justified this in Section 3 for the high-energy limit), one easily gets

$$
\epsilon_{T T T}: \epsilon_{(L L T)}: \epsilon_{(L T)}: \epsilon_{[L T]}=8: 1: 3:-3 .
$$

After including the normalization factor of the field variables ${ }^{4}$ and the appropriate symmetry factors, one ends up with

$$
\begin{aligned}
& \mathcal{T}_{T T T}: \mathcal{T}_{(L L T)}: \mathcal{T}_{(L T)}: \mathcal{T}_{[L T]} \\
& \quad=6 \epsilon_{T T T}: 6 \epsilon_{(L L T)}:-2 \epsilon_{(L T)}:-2 \epsilon_{[L T]}=8: 1:-1: 1 .
\end{aligned}
$$

Here the definitions of $\mathcal{T}_{T T T}, \mathcal{T}_{(L L T)}, \mathcal{T}_{(L T)}, \mathcal{T}_{[L T]}$ and similar amplitudes hereafter can be found in $[12,13]$ and the result obtained is consistent with the previous zero-norm state calculation in [12] or Eq. (13).

\subsection{2. $m^{2}=6$}

The most general form of physical states at mass level $m^{2}=6$ are given by

$$
\begin{aligned}
& {\left[\epsilon_{\mu \nu \lambda \sigma} \alpha_{-1}^{\mu} \alpha_{-1}^{\nu} \alpha_{-1}^{\lambda} \alpha_{-1}^{\sigma}+\epsilon_{(\mu \nu \lambda)} \alpha_{-1}^{\mu} \alpha_{-1}^{\nu} \alpha_{-2}^{\lambda}+\epsilon_{\mu \nu, \lambda} \alpha_{-1}^{\mu} \alpha_{-1}^{\nu} \alpha_{-2}^{\lambda}\right.} \\
& \left.+\epsilon_{(\mu \nu)}^{(1)} \alpha_{-1}^{\mu} \alpha_{-3}^{\nu}+\epsilon_{[\mu \nu]}^{(1)} \alpha_{-1}^{\mu} \alpha_{-3}^{\nu}+\epsilon_{(\mu \nu)}^{(2)} \alpha_{-2}^{\mu} \alpha_{-2}^{\nu}+\epsilon_{\mu} \alpha_{-4}^{\mu}\right]|0, k\rangle,
\end{aligned}
$$

where $\epsilon_{\mu \nu, \lambda}$ represents the mixed symmetric spin three states, that is, one first symmetrizes $\mu \nu$ and then anti-symmetrizes $\mu \lambda$. The Virasoro constraints are calculated to be

$$
2 k^{\sigma} \epsilon_{(\mu \nu \lambda \sigma)}+\epsilon_{(\mu \nu \lambda)}=0
$$

\footnotetext{
4 The normalization factors are determined by the inner product of a state with its BPZ conjugate.
} 


$$
\begin{aligned}
& 2 k^{\lambda} \epsilon_{(\mu \nu \lambda)}+k^{\lambda}\left(\epsilon_{\lambda \mu, v}+\epsilon_{\mu \lambda, v}\right)+3\left(\epsilon_{(\mu \nu)}^{(1)}+\epsilon_{[\mu \nu]}^{(1)}\right)+4 \epsilon_{(\mu \nu)}^{(2)}=0, \\
& k^{\mu} \epsilon_{(\mu \nu)}^{(1)}+k^{\mu} \epsilon_{[\mu \nu]}^{(1)}+4 \epsilon_{v}=0, \\
& 6 \eta^{\lambda \sigma} \epsilon_{(\mu \nu \lambda \sigma)}+2 k^{\lambda} \epsilon_{(\mu \nu \lambda)}+\frac{1}{2} k^{\lambda}\left(\epsilon_{\mu \nu, \lambda}+\epsilon_{\nu \mu, \lambda}\right)+3 \epsilon_{(\mu \nu)}^{(1)}=0, \\
& \eta^{\mu \nu} \epsilon_{(\mu \nu \lambda)}+\eta^{\mu \nu} \epsilon_{(\mu \nu, \lambda)}+4 k^{\mu} \varepsilon_{(\mu \nu)}^{(2)}+4 \epsilon_{\lambda}=0 .
\end{aligned}
$$

In the high-energy limit, similar calculation as above gives

$$
\begin{aligned}
& \mathcal{T}_{(T T T T)}: \mathcal{T}_{(T T L L)}: \mathcal{T}_{(L L L L)}: \mathcal{T}_{T T, L}: \mathcal{T}_{(T T L)}: \mathcal{T}_{(L L L)}: \mathcal{T}_{(L L)} \\
& \quad=4 ! \epsilon_{(T T T T)}: 4 ! \epsilon_{(T T L L)}: 4 ! \epsilon_{(L L L L)}:-4 \epsilon_{T T, L}:-4 \epsilon_{(T T L)}:-4 \epsilon_{(L L L)}: 8 \epsilon_{(L L)}^{(2)} \\
& \quad=16: \frac{4}{3}: \frac{1}{3}:-\frac{2 \sqrt{6}}{3}:-\frac{4 \sqrt{6}}{9}:-\frac{\sqrt{6}}{9}: \frac{2}{3},
\end{aligned}
$$

which is consistent with the previous zero-norm state calculation in [13] or Eq. (13).

\subsection{3. $m^{2}=8$}

The most general form of physical states at mass level $m^{2}=8$ are given by (for simplicity, we neglect terms containing $\alpha_{-n}^{\mu}$ with $n \geqslant 3$ )

$$
\begin{aligned}
& {\left[\epsilon_{\mu \nu \lambda \sigma \rho} \alpha_{-1}^{\mu} \alpha_{-1}^{\nu} \alpha_{-1}^{\lambda} \alpha_{-1}^{\sigma} \alpha_{-1}^{\rho}+\epsilon_{(\mu \nu \lambda \sigma)} \alpha_{-1}^{\mu} \alpha_{-1}^{\nu} \alpha_{-1}^{\lambda} \alpha_{-2}^{\rho}+\epsilon_{(\mu \nu \lambda)} \alpha_{-1}^{\mu} \alpha_{-2}^{\nu} \alpha_{-2}^{\lambda}\right.} \\
& \left.\quad+\epsilon_{\mu \nu \lambda, \sigma} \alpha_{-1}^{\mu} \alpha_{-1}^{\nu} \alpha_{-1}^{\lambda} \alpha_{-2}^{\rho}+\epsilon_{\mu, \nu \lambda} \alpha_{-1}^{\mu} \alpha_{-2}^{\nu} \alpha_{-2}^{\lambda}\right]|0, k\rangle
\end{aligned}
$$

where $\epsilon_{\mu \nu \lambda, \sigma}$ represents the mixed symmetric spin four states, that is, first symmetrizes $\mu \nu \lambda$ and then anti-symmetrizes $\mu \sigma$. Similar definition for the mixed symmetric spin three states $\epsilon_{\mu, \nu \lambda}$. The Virasoro constraints are calculated to be

$$
\begin{aligned}
& 5 k^{\sigma} \epsilon_{(\mu \nu \lambda \sigma \rho)}+2 \epsilon_{(\mu \nu \lambda \sigma)}=0, \\
& 3 k^{\lambda} \epsilon_{(\mu \nu \lambda \sigma)}+\frac{1}{2} k^{\lambda}\left[\left(\epsilon_{\mu \nu \lambda, \sigma}+\epsilon_{\lambda \mu \nu, \sigma}+\epsilon_{\mu \lambda \nu, \sigma}\right)+(\mu \leftrightarrow v)\right] \\
& \quad+4 \epsilon_{(\mu \nu \sigma)}+\epsilon_{\mu, \nu \sigma}+\epsilon_{\nu, \mu \sigma}=0, \\
& k^{\mu} \epsilon_{(\mu \nu \lambda)}+\frac{1}{2} k^{\mu}\left(\epsilon_{\mu, \nu \lambda}+\epsilon_{\mu, \lambda \nu}\right)=0, \\
& 5 \eta^{\rho \sigma} \epsilon_{(\mu \nu \lambda \sigma \rho)}+k^{\sigma} \epsilon_{(\mu \nu \lambda \sigma)}+\frac{1}{3} k^{\sigma}\left(\epsilon_{\mu \nu \lambda, \sigma}+\epsilon_{\nu \lambda \mu, \sigma}+\epsilon_{\lambda \mu \nu, \sigma}\right)=0, \\
& 3 \eta^{\nu \lambda} \epsilon_{(\mu \nu \lambda \sigma)}+\eta^{\nu \lambda}\left(\epsilon_{\mu \nu \lambda, \sigma}+\epsilon_{\lambda \mu \nu, \sigma}+\epsilon_{\nu \lambda \mu, \sigma}\right)+4 k^{\lambda} \varepsilon_{(\mu \sigma \lambda)} \\
& \quad+2 k^{\lambda}\left(\varepsilon_{\mu, \sigma \lambda}+\varepsilon_{\mu, \lambda \sigma}\right)=0 .
\end{aligned}
$$

In the high-energy limit, similar calculation as above gives

$$
\begin{aligned}
& \mathcal{T}_{(T T T T T)}: \mathcal{T}_{(T T T L)}: \mathcal{T}_{(T T T L L)}: \mathcal{T}_{(T L L L)}: \mathcal{T}_{(T L L L L)}: \mathcal{T}_{(T L L)}: \mathcal{T}_{T, L L}: \mathcal{T}_{T L L, L}: \mathcal{T}_{T T T, L} \\
&= 5 ! \epsilon_{(T T T T T)}: 3 ! \times 2 \epsilon_{(T T T L)}: 5 ! \epsilon_{(T T T L L)}: 3 ! \times 2 \epsilon_{(T L L L)}: 5 ! \epsilon_{(T L L L L)} \\
&: 8 \epsilon_{(T L L)}: 8 \epsilon_{T, L L}: 3 ! \times 2 \epsilon_{T L L, L}: 3 ! \times 2 \epsilon_{T T T, L} \\
&= 32: \sqrt{2}: 2: \frac{3 \sqrt{2}}{16}: \frac{3}{8}: \frac{1}{3}: \frac{2}{3}: \frac{\sqrt{2}}{16}: 3 \sqrt{2}
\end{aligned}
$$


which can be checked to be remarkably consistent with the results of Eq. (13) after Young tableaux decomposition.

\subsection{General mass levels}

In this section we calculate the ratios of string scattering amplitudes in the high-energy limit for general mass levels by imposing Virasoro constraints. The final result will, of course, be exactly the same as what we obtained by requiring the decoupling of highenergy zero-norm states. In the presentation here we use the notation of Young's tableaux.

We consider the general mass level $m^{2}=2(n-1)$. The most general state can be written as

$$
|n\rangle=\left\{\sum_{m_{j}} \bigotimes_{j=1}^{k} \frac{1}{j^{m_{j} m_{j} !}} \begin{array}{|l|l|l|}
\mu_{1}^{j} & \cdots & \mu_{m_{j}}^{j} \\
\alpha_{-j}^{\mu_{1}^{j} \cdots \mu_{m_{j}}^{j}}
\end{array}\right\}|0, k\rangle,
$$

where we defined the abbreviation

$$
\alpha_{-j}^{\mu_{1}^{j} \cdots \mu_{m_{j}}^{j}} \equiv \alpha_{-j}^{\mu_{1}^{j}} \cdots \alpha_{-j}^{\mu_{m_{j}}^{j}}
$$

with $m_{j}$ is the number of the operator $\alpha_{-j}^{\mu}$. The summation runs over all possible combinations of $m_{j}$ 's with the constraints

$$
\sum_{j=1}^{k} j m_{j}=n \quad \text { and } \quad 0 \leqslant m_{j} \leqslant n
$$

so that the total mass is $n$. It is obvious that $k$ is less or equal to $n$. Since the upper indices $\left\{\mu_{1}^{j} \cdots \mu_{m_{j}}^{j}\right\}$ in $\alpha_{-j}^{\mu_{1}^{j}} \cdots \alpha_{-j}^{\mu_{m_{j}}^{j}}$ are symmetric, we used the Young tableaux notation to denote the coefficients in Eq. (56). The direct product $\otimes$ acts on the Young tableaux in the standard way, for example,

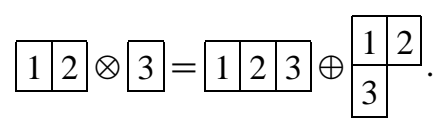

Finally, $1 /\left(j^{m_{j}} m_{j} !\right)$ are the normalization factors. To be clear, for example $n=4$, the state can be written as

$$
\begin{aligned}
|4\rangle= & \left\{\frac{1}{4 !} \mu_{1}^{1} \mu_{2}^{1} \mu_{3}^{1} \mu_{4}^{1} \alpha_{-1}^{\mu_{1}^{1}} \alpha_{-1}^{\mu_{2}^{1}} \alpha_{-1}^{\mu_{3}^{1}} \alpha_{-1}^{\mu_{4}^{1}}+\frac{1}{2 \cdot 2 !} \mu_{1}^{1} \mu_{2}^{1} \otimes \mu_{1}^{2} \alpha_{-1}^{\mu_{1}^{1}} \alpha_{-1}^{\mu_{2}^{1}} \alpha_{-2}^{\mu_{1}^{2}}\right. \\
& +\frac{1}{3} \mu_{1}^{1} \otimes \mu_{1}^{3} \alpha_{-1}^{\mu_{1}^{1}} \alpha_{-3}^{\mu_{1}^{3}}+\frac{1}{2^{2} \cdot 2 !} \mu_{1}^{2} \mu_{2}^{2} \alpha_{-2}^{\mu_{1}^{2}} \alpha_{-2}^{\mu_{2}^{2}}+\frac{1}{4} \mu_{1}^{4} \alpha_{-4}^{\mu_{1}^{4}}|0, k\rangle .
\end{aligned}
$$

Next, we will apply the Virasoro constraints to the state Eq. (56). The only Virasoro constraints which need to be considered are

$$
L_{1}|n\rangle=L_{2}|n\rangle=0
$$


with $L_{m}$ the standard Virasoro operator

$$
L_{m}=\frac{1}{2} \sum_{n=-\infty}^{\infty} \alpha_{m+n} \cdot \alpha_{-n} .
$$

After taking care the symmetries of the Young tableaux, the Virasoro constraints become

$$
\begin{aligned}
& L_{1}|n\rangle=\sum_{m_{j}}\left[k^{\mu_{1}^{1}} \bigotimes_{j=1}^{k} \mu_{1}^{j} \mid \cdots \mu_{m_{j}}^{j}\right. \\
& +\sum_{i=2}^{m_{1}} \begin{array}{|l|l|l|l|l|}
\hline \mu_{2}^{1} & \cdots & \hat{\mu}_{i}^{1} & \cdots & \mu_{m_{1}}^{1}
\end{array} \otimes \begin{array}{|l|l|l|l|}
\hline \mu_{i}^{1} & \mu_{1}^{2} & \cdots & \mu_{m_{2}}^{2}
\end{array} \bigotimes_{j \neq 1,2}^{k} \begin{array}{|l|l|l|}
\mu_{1}^{j} & \cdots & \mu_{m_{j}}^{j} \\
\hline
\end{array} \\
& +\sum_{l=3}^{k}(l-1) \begin{array}{|l|l|l|}
\mu_{2}^{1} & \cdots & \mu_{m_{1}}^{1}
\end{array} \otimes \sum_{i=1}^{m_{l-1}} \begin{array}{|l|l|l|l|l|}
\hline \mu_{1}^{l-1} & \cdots & \hat{\mu}_{i}^{l-1} & \cdots & \mu_{m_{l-1}}^{l-1} \\
\hline
\end{array} \\
& \otimes \begin{array}{|l|l|l|l|}
\hline \mu_{i}^{l-1} & \mu_{1}^{l} & \cdots & \mu_{m_{l}}^{l}
\end{array} \bigotimes_{j \neq 1, l, l-1}^{k} \begin{array}{|l|l|l|}
\hline \mu_{1}^{j} & \cdots & \mu_{m_{j}}^{j} \\
\hline
\end{array} \frac{1}{\left(m_{1}-1\right) !} \alpha_{-1}^{\mu_{2}^{1} \cdots \mu_{m_{1}}^{1}} \\
& \times \prod_{j \neq 1}^{k} \frac{1}{j^{m_{j}} m_{j} !} \alpha_{-j}^{\mu_{1}^{j} \cdots \mu_{m_{j}}^{j}}|0, k\rangle=0,
\end{aligned}
$$

and

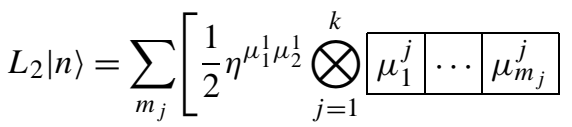

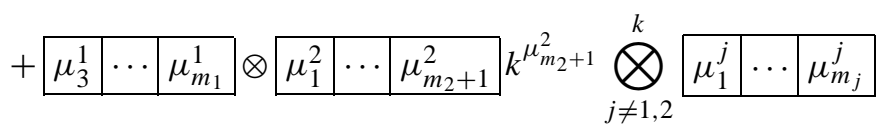

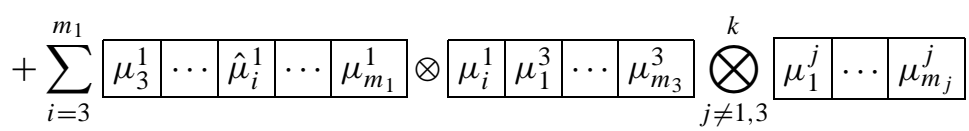

$$
\begin{aligned}
& +\sum_{l=4}^{k}(l-2) \begin{array}{|l|l|l|}
\mu_{3}^{1} & \cdots & \mu_{m_{1}}^{1}
\end{array} \otimes \sum_{i=1}^{m_{l-2}} \begin{array}{|l|l|l|l|l|}
\mu_{1}^{l-2} & \cdots & \hat{\mu}_{i}^{l-2} & \cdots & \mu_{m_{l}}^{l-2} \\
\hline
\end{array} \\
& \left.\otimes \begin{array}{|l|l|l|l|l}
\hline \mu_{i}^{l-2} & \mu_{1}^{l} & \cdots & \mu_{m_{l}}^{l}
\end{array} \bigotimes_{j \neq 1, l, l-2} \begin{array}{|l|l|l|}
\mu_{1}^{j} & \cdots & \mu_{m_{j}}^{j} \\
\hline
\end{array}\right] \frac{1}{\left(m_{1}-2\right) !} \alpha_{-1}^{\mu_{3}^{1} \cdots \mu_{m_{1}}^{1}} \\
& \times \prod_{j \neq 1}^{k} \alpha_{-j}^{\mu_{1}^{j} \cdots \mu_{m}^{j}}|0, k\rangle=0
\end{aligned}
$$


A hat on an index means that the index is skipped there (and it should appear somewhere else). In the above derivation we have used the identity for the Young tableaux

$$
\begin{aligned}
& \begin{array}{|l|l|l|}
\hline 1 & \cdots & p
\end{array}=\frac{1}{p}\left[1+\sigma_{(21)}+\sigma_{(321)}+\cdots+\sigma_{(p \cdots 1)}\right] \begin{array}{|l|l|l|}
2 & \cdots & p
\end{array} 1 \\
& =\frac{1}{p} \sum_{i=1}^{p} \sigma_{(i 1)} \begin{array}{|l|l|l|}
2 & \cdots & p
\end{array} \quad \begin{array}{l}
1 \\
\hline
\end{array}
\end{aligned}
$$

where $\sigma_{(i \cdots j)}$ are permutation operators.

States which satisfy the Virasoro constraints are physical states. What we are going to show in the following is that, in the high-energy limit, the Virasoro constraints turn out to be strong enough to give the linear relationship among the physical states. To take the high-energy limit in the above equations (63a) and (63b), we replace the indices $\left(\mu_{i}, v_{i}\right)$ by $L$ or $T$, and

$$
k^{\mu_{i}} \rightarrow \hat{m} e^{L}, \quad \eta^{\mu_{1} \mu_{2}} \rightarrow e^{T} e^{T},
$$

where $\hat{m}$ is the mass operator. The Virasoro constraints at high energy are derived in Appendix C.1 as Eqs. (C.4a) and (C.4b). To solve the constraints, we need the following lemma to further simplify them.

\section{Lemma.}

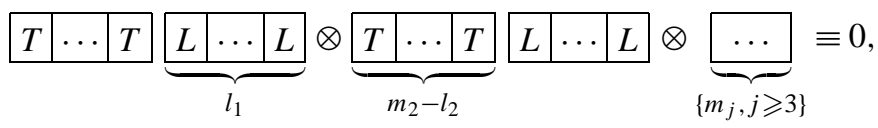

except for (i) $l_{2}=m_{2}, m_{j}=0$ for $j \geqslant 3$ and (ii) $l_{1}=2 m$.

This lemma is equivalent to part of the results of Section 3, but will also be proved in Appendix C.2 by applying Virasoro constraints. Finally, the Virasoro constraints at highenergy reduce to

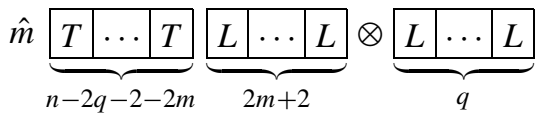

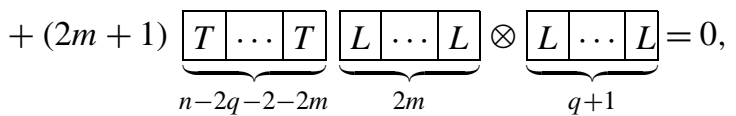

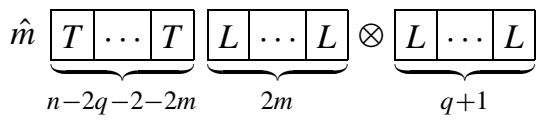

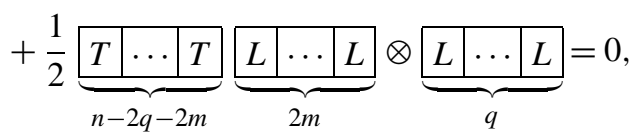

where we have renamed $m_{2} \rightarrow q$ and $m_{1} \rightarrow n-2 q$. 
By mathematical recursion, Eq. (67a) leads to

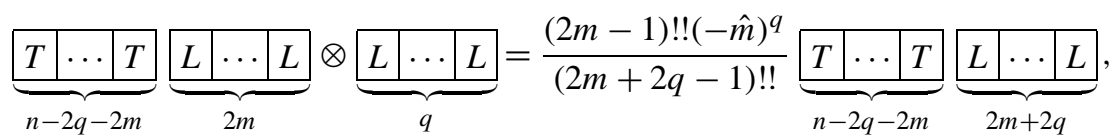

and similarly, Eq. (67b) leads to

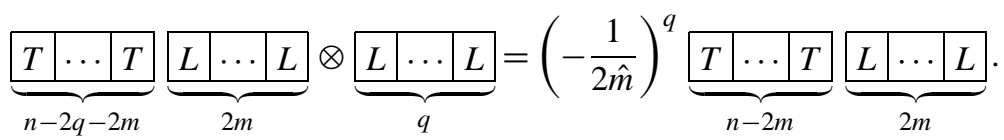

Combining Eqs. (68a) and (68b), we get

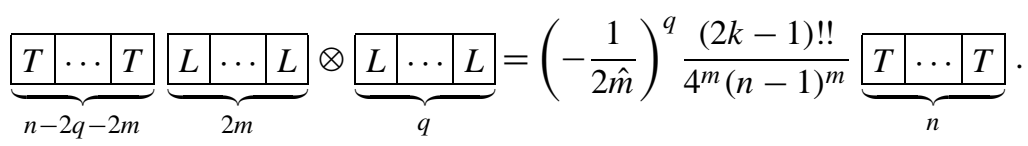

This is equivalent to Eq. (13).

To get the ratio for the specific physical states, we make the Young tableaux decomposition

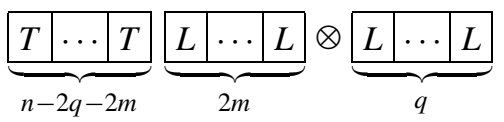

$$
\begin{aligned}
& =\sum_{l=0}^{\min \{n-2 q-2 m, q\}} \begin{array}{|l|l|l|l|l|l|l|l|}
T & \multicolumn{2}{|c}{\cdots} & T & L & \cdots & L \\
\hline L & \cdots & L & & & &
\end{array} \cdot\left(l ! C_{q}^{l} C_{n-2 q-2 m}^{l}\right),
\end{aligned}
$$

where $C_{q}^{l}=\frac{q !}{l !(q-l) !}$ and we have $(n-2 q-2 m) T$ 's and $(2 m+q-l) L$ 's in the first column, $(l) L$ 's in the second column in the second line of the above equation. Therefore, we obtain

$$
\begin{aligned}
& \begin{array}{|l|l|l|l|l|l|l|l|}
\hline T & \multicolumn{3}{|c|}{\cdots} & T & L & \cdots & L \\
\hline L & \cdots & L & & &
\end{array} \cdot\left(l ! C_{q}^{l} C_{n-2 q-2 m}^{l}\right) \\
& =\frac{l ! C_{q}^{l} C_{n-2 q-2 m}^{l}}{\sum_{l=0}^{\min \{n-2 q-2 m, q\}} l ! C_{q}^{l} C_{n-2 q-2 m}^{l}}\left(-\frac{1}{2 \hat{m}}\right)^{q} \frac{(2 m-1) ! !}{4^{m}(n-1)^{m}} \underbrace{\underbrace{}_{n} \ldots T}_{n},
\end{aligned}
$$

which is consistent with the ratios Eqs. (41), (48) and (55) for $m^{2}=4,6$, 8, respectively.

\section{Saddle-point approximation for stringy amplitudes}

In previous sections, we have identified the leading high-energy amplitudes and derived the ratios among high-energy amplitudes for members of a family at given mass levels, based on decoupling principle. While deductive arguments help to clarify the underlying assumptions and solidify the validity of decoupling principle, it is instructive to compare 
it with a different approach, such as the saddle-point approximation [14]. Therefore, we shall perform direct calculations to check the results obtained above and make comparisons between these two approaches.

In this section, we give a direct verification of the ratios among leading high-energy amplitudes based on the saddle-point method. The four-point amplitudes to be calculated consist of one massive tensor and three tachyons. Since we have shown that in the highenergy limit the only relevant states are those corresponding to

$$
\left(\alpha_{-1}^{T}\right)^{n-2 m-2 q}\left(\alpha_{-1}^{L}\right)^{2 m}\left(\alpha_{-2}^{L}\right)^{q}|0, k\rangle, \quad-k^{2}=2(n-1),
$$

we only need to calculate the following four-point amplitude

$$
\mathcal{T}^{(n, 2 m, q)} \equiv \int \prod_{i=1}^{4} d x_{i}\left\langle V_{1} V_{2}^{(n, 2 m, q)} V_{3} V_{4}\right\rangle,
$$

where

$$
\begin{aligned}
& V_{2}^{(n, 2 m, q)} \equiv\left(\partial X^{T}\right)^{n-2 m-2 q}\left(\partial X^{P}\right)^{2 m}\left(\partial^{2} X^{P}\right)^{q} e^{i k_{2} X_{2}}, \\
& V_{i} \equiv e^{i k_{i} X_{i}}, \quad i=1,3,4 .
\end{aligned}
$$

Notice that here for leading high-energy amplitudes we replace the polarization $L$ by $P$.

Using either path-integral or operator formalism, after $\operatorname{SL}(2, R)$ gauge fixing, we obtain the $s-t$ channel contribution to the stringy amplitude at tree level

$$
\begin{aligned}
\mathcal{T}^{(n, 2 m, q)} \Rightarrow & \int_{0}^{1} d x x^{(1,2)}(1-x)^{(2,3)}\left[\frac{e^{T} \cdot k_{1}}{x}-\frac{e^{T} \cdot k_{3}}{1-x}\right]^{n-2 m-2 q} \\
& \times\left[\frac{e^{P} \cdot k_{1}}{x}-\frac{e^{P} \cdot k_{3}}{1-x}\right]^{2 m}\left[-\frac{e^{P} \cdot k_{1}}{x^{2}}-\frac{e^{P} \cdot k_{3}}{(1-x)^{2}}\right]^{q},
\end{aligned}
$$

where we have simplified the inner products among momenta by defining $(1,2) \equiv k_{1} \cdot k_{2}$.

In order to apply the saddle-point method, we need to rewrite the amplitude above into the "canonical form". That is,

$$
\mathcal{T}^{(n, 2 m, q)}(K)=\int_{0}^{1} d x u(x) e^{-K f(x)},
$$

where

$$
\begin{aligned}
& K \equiv-(1,2) \rightarrow \frac{s}{2} \rightarrow 2 E^{2}, \\
& \tau \equiv-\frac{(2,3)}{(1,2)} \rightarrow-\frac{t}{s} \rightarrow \sin ^{2} \frac{\phi}{2}, \\
& f(x) \equiv \ln x-\tau \ln (1-x), \\
& u(x) \equiv\left[\frac{(1,2)}{\hat{m}}\right]^{2 m+q}(1-x)^{-n+2 m+2 q}\left(f^{\prime}\right)^{2 m}\left(f^{\prime \prime}\right)^{q}\left(-e^{T} \cdot k_{3}\right)^{n-2 m-2 q} .
\end{aligned}
$$


The saddle-point for the integration of moduli, $x=x_{0}$, is defined by

$$
f^{\prime}\left(x_{0}\right)=0,
$$

and we have

$$
x_{0}=\frac{1}{1-\tau}, \quad 1-x_{0}=-\frac{\tau}{1-\tau}, \quad f^{\prime \prime}\left(x_{0}\right)=(1-\tau)^{3} \tau^{-1} .
$$

From the definition of $u(x)$, it is easy to see that

$$
u\left(x_{0}\right)=u^{\prime}\left(x_{0}\right)=\cdots=u^{(2 m-1)}\left(x_{0}\right)=0,
$$

and

$$
u^{(2 m)}\left(x_{0}\right)=\left[\frac{(1,2)}{\hat{m}}\right]^{2 m+q}\left(1-x_{0}\right)^{-n+2 m+2 q}(2 m) !\left(f_{0}^{\prime \prime}\right)^{2 m+q}\left(-e^{T} \cdot k_{3}\right)^{n-2 m-2 q} .
$$

With these inputs, one can easily evaluate the Gaussian integral associated with the four-point amplitudes, Eq. (77),

$$
\begin{array}{rl}
\int_{0}^{1} & d x u(x) e^{-K f(x)} \\
& =\sqrt{\frac{2 \pi}{K f_{0}^{\prime \prime}}} e^{-K f_{0}}\left[\frac{u_{0}^{(2 m)}}{2^{m} m !\left(f_{0}^{\prime \prime}\right)^{m} K^{m}}+O\left(\frac{1}{K^{m+1}}\right)\right] \\
& =\sqrt{\frac{2 \pi}{K f_{0}^{\prime \prime}}} e^{-K f_{0}}\left[(-1)^{n-q} \frac{2^{n-2 m-q}(2 m) !}{m ! \hat{m}^{2 m+q}} \tau^{-\frac{n}{2}}(1-\tau)^{\frac{3 n}{2}} E^{n}+O\left(E^{n-2}\right)\right] .
\end{array}
$$

This result shows explicitly that with one tensor and three tachyons, the energy and angle dependence for the high-energy four-point amplitudes only depend on the level $n$, and we can solve for the ratios among high-energy amplitudes within the same family,

$$
\begin{aligned}
\lim _{E \rightarrow \infty} \frac{\mathcal{T}^{(n, 2 m, q)}}{\mathcal{T}^{(n, 0,0)}} & =\frac{(-1)^{q}(2 m) !}{m !(2 \hat{m})^{2 m+q}} \\
& =\left(-\frac{2 m-1}{\hat{m}}\right) \cdots\left(-\frac{3}{\hat{m}}\right)\left(-\frac{1}{\hat{m}}\right)\left(-\frac{1}{2 \hat{m}}\right)^{m+q},
\end{aligned}
$$

which is consistent with Eq. (13).

We conclude this section with three remarks. Firstly, from the saddle-point approach, it is easy to see why the product of $\alpha_{-1}^{P}$ oscillators induce energy suppression. Their contribution to the stringy amplitude is proportional to powers of $f^{\prime}\left(x_{0}\right)$, which is zero in the leading order calculation. Secondly, one can also understand why only even numbers of $\alpha_{-1}^{P}$ oscillators will survive for high-energy amplitudes based on the structure of Gaussian integral in Eq. (77). While for a vertex operator containing $(2 m+1) \alpha_{-1}^{P}$ 's, we have $u\left(x_{0}\right)=u^{\prime}\left(x_{0}\right)=\cdots=u^{(2 m)}\left(x_{0}\right)=0$, and the leading contribution comes from $u^{(2 m+1)}\left(x_{0}\right)\left(x-x_{0}\right)^{2 m+1}$, this gives zero since the odd-power moments of Gaussian integral vanish. Finally, for the alert readers, since we only discuss the $s-t$ channel contribution 
to the scattering amplitudes, the integration range for the $x$ variable seems to devoid of a direct application of saddle-point method. Presumably, we can apply the saddle-point approximation to the full amplitudes whose integration range extends over whole real line. However, it is curious to see why $s-t$ channel alone has the same functional form as the full amplitude in the high-energy limit. To see this, one can check that the leading contribution Eq. (86) is actually "form-invariant" under any monotonous change of variable $x=\xi(y), v(y) \equiv \xi^{\prime}(y) u(\xi(y)), g(y) \equiv f(\xi(y))$. If the analytic structure of the integrand is no concern, we can justify the use of saddle-point approximation even for the case of $s-t$ channel.

\section{Remarks}

\subsection{Virasoro generators at high energies}

In retrospect, the main result (13) can be very easily obtained from the Virasoro generators if we accept that we only need to consider states of the form

$$
\left(\alpha_{-1}^{T}\right)^{n-m-2 q}\left(\alpha_{-1}^{L}\right)^{m}\left(\alpha_{-2}^{L}\right)^{q}|0, k\rangle .
$$

On the space of these states, the Virasoro generators are effectively

$$
\begin{aligned}
& \tilde{L}_{-1}=\hat{m} \alpha_{-1}^{L}+\alpha_{-2}^{L} \alpha_{1}^{L}, \\
& \tilde{L}_{-2}=\hat{m} \alpha_{-2}^{L}+\frac{1}{2} \alpha_{-1}^{T} a_{-1}^{T}, \\
& \tilde{L}_{-n}=0 \quad \text { for } n \geqslant 3,
\end{aligned}
$$

where we have also replaced $e^{P}$ by $e^{L}$.

Using these deformed Virasoro generators, we create high-energy approximations of spurious states. On the back of an envelope, one can check that the decoupling of the spurious states created by $\tilde{L}_{-1}$ and $\tilde{L}_{-2}$ implies (32) and (35), respectively (with $P$ replaced by $L$ ). In short, $\tilde{L}_{-1}$ tells us how to trade $\alpha_{-1}^{L} \alpha_{-1}^{L}$ for $\alpha_{-2}^{L}$, and $\tilde{L}_{-2}$ tells us how to trade $\alpha_{-2}^{L}$ for $\alpha_{-1}^{T} \alpha_{-1}^{T}$.

Similarly, the Hermitian conjugates of $\tilde{L}_{1}$ and $\tilde{L}_{2}$ can be used to derive the same result by demanding that they annihilate states in the high-energy limit.

\subsection{2-dimensional string}

Although we have shown that there exist infinitely many linear relations among 4-point functions which uniquely fix their ratios in the high-energy limit, it is not totally clear that there is a hidden symmetry responsible for it. However, we would like to claim that these linear relations are indeed the manifestation of the long-sought hidden symmetry of string theory, and that we are on the right track of understanding the symmetry. To persuade the readers, we test our claim on a toy model of string theory - the 2-dimensional string theory.

While the hidden symmetry of the 26-dimensional bosonic string theory is still at large, the symmetry of the 2-dimensional string theory is much better understood. It is known to 
be associated with the discrete states

$$
\psi_{J M}^{ \pm} \sim \Delta(J, M,-i \sqrt{2} X) \exp [\sqrt{2}(i M X(0)+( \pm J-1) \phi(0))]
$$

Half of them $\psi_{J M}^{+}$generate the $w_{\infty}$ algebra [16]

$$
\int \frac{d z}{2 \pi i} \psi_{J_{1} M_{1}}^{+} \psi_{J_{2} M_{2}}^{+} \sim\left(J_{2} M_{1}-J_{1} M_{2}\right) \psi_{\left(J_{1}+J_{2}-1\right)\left(M_{1}+M_{2}\right)}^{+}
$$

Let us now check whether the $w_{\infty}$ symmetry is generated by the high-energy limit of zero-norm states. In [15], explicit expression for a class of zero-norm states was given

$$
\begin{aligned}
G_{J M}^{+} \sim & (J-M) ! \Delta(J, M,-i \sqrt{2} X) \exp [\sqrt{2}(i M X+(J-1) \phi] \\
& +(-1)^{2 J} \sum_{j=1}^{J-M}(J-M-1) ! \int \frac{d z}{2 \pi i} \mathcal{D}(J, M,-i \sqrt{2} X(z), j) \\
& \times \exp [\sqrt{2}(i(M+1) X(z)+(J-1) \phi(z)-X(0))] .
\end{aligned}
$$

The notation needs some explanation. Here $\Delta(J, M,-i \sqrt{2} X)$ is defined by

$$
\Delta(J, M,-i \sqrt{2} X)=\left|\begin{array}{cccc}
S_{2 J-1} & S_{2 J-2} & \cdots & S_{J+M} \\
S_{2 J-2} & S_{2 J-3} & \cdots & S_{J+M-1} \\
\cdots & \cdots & \cdots & \cdots \\
S_{J+M} & S_{J+M-1} & \cdots & S_{2 M+1}
\end{array}\right|,
$$

where

$$
S_{k}=S_{k}\left(\left\{\frac{-i \sqrt{2}}{k !} \partial^{k} X(0)\right\}\right), \quad \text { and } \quad S_{k}=0 \quad \text { if } k<0,
$$

and $S_{k}\left(\left\{a_{i}\right\}\right)$ 's denote the Schur polynomial defined by

$$
\exp \left(\sum_{k=1}^{\infty} a_{k} x^{k}\right)=\sum_{k=0}^{\infty} S_{k}\left(\left\{a_{i}\right\}\right) x^{k}
$$

$\mathcal{D}(J, M,-i \sqrt{2} X(z), j)$ is defined by a similar expression as Eq. (95), but with the $j$ th row replaced by $\left\{(-z)^{j}-1-2 J,(-z)^{j}-2 J, \ldots,(-z)^{j-J-M-2}\right\}$. It was shown [15] that zero-norm states in Eq. (94) generate a $w_{\infty}$ algebra.

In the high-energy limit, the factors $\partial^{k} X^{A}$ are generically proportional to a linear combination of the momenta of other vertices, so it scales with energy $E$. Thus $\mathcal{D}(J, M,-i \sqrt{2} X, j)$ is subleading to $\Delta(J, M,-i \sqrt{2} X)$. Ignoring the second term in Eq. (94) for this reason, we see that these zero-norm states indeed approach to the discrete states $\psi_{J M}^{+}$above! Thus, the $w_{\infty}$ algebra generated by Eq. (94) is identified to $w_{\infty}$ symmetry in Eq. (93). This result strongly suggests that the linear relations among correlation functions obtained from HZNS are indeed related to the hidden symmetry also for the 26-dimensional strings. Although we still do not know what is the symmetry group, or how it acts on states, this work sheds new light on the road to finding the answers. 


\section{Acknowledgement}

The authors thank Jiunn-Wei Chen, Tohru Eguchi, Koji Hashimoto, Hiroyuki Hata, Takeo Inami, Yeong-Chuan Kao, Yoichi Kazama, Yutaka Matsuo, Tamiaki Yoneya for helpful discussions. This work is supported in part by the National Science Council and the National Center for Theoretical Sciences, Taiwan, ROC.

\section{Appendix A. Kinematic variables and notations}

For the readers' convenience, we list the expressions of the kinematic variables involved in the evaluation of a 4-point function in this appendix. In Fig. 1, we take the scattering plane to be the $X^{1}-X^{2}$ plane. The momenta of the particles are

$$
\begin{aligned}
& k_{1}=\left(\sqrt{p^{2}+m_{1}^{2}},-p, 0\right), \\
& k_{2}=\left(\sqrt{p^{2}+m_{2}^{2}}, p, 0\right), \\
& k_{3}=\left(-\sqrt{q^{2}+m_{3}^{2}},-q \cos \phi,-q \sin \phi\right), \\
& k_{4}=\left(-\sqrt{q^{2}+m_{4}^{2}}, q \cos \phi, q \sin \phi\right) .
\end{aligned}
$$

They satisfy $k_{i}^{2}=-m_{i}^{2}$. In the high-energy limit, the Mandelstam variables are

$$
\begin{aligned}
& s \equiv-\left(k_{1}+k_{2}\right)^{2}=4 E^{2}+\mathcal{O}\left(1 / E^{2}\right), \\
& t \equiv-\left(k_{2}+k_{3}\right)^{2}=-4\left(E^{2}-\frac{\sum_{i=1}^{4} m_{i}^{2}}{4}\right) \sin ^{2} \frac{\phi}{2}+\mathcal{O}\left(1 / E^{2}\right), \\
& u \equiv-\left(k_{1}+k_{3}\right)^{2}=-4\left(E^{2}-\frac{\sum_{i=1}^{4} m_{i}^{2}}{4}\right) \cos ^{2} \frac{\phi}{2}+\mathcal{O}\left(1 / E^{2}\right),
\end{aligned}
$$

where $E$ is related to $p$ and $q$ as

$$
E^{2}=p^{2}+\frac{m_{1}^{2}+m_{2}^{2}}{2}=q^{2}+\frac{m_{3}^{2}+m_{4}^{2}}{2} .
$$

The polarization bases for the 4 particles are

$$
\begin{aligned}
& e^{L}(1)=\frac{1}{m_{1}}\left(p,-\sqrt{p^{2}+m_{1}^{2}}, 0\right), \quad e^{T}(1)=(0,0,-1), \\
& e^{L}(2)=\frac{1}{m_{2}}\left(p, \sqrt{p^{2}+m_{2}^{2}}, 0\right), \quad e^{T}(2)=(0,0,1), \\
& e^{L}(3)=\frac{1}{m_{3}}\left(-q,-\sqrt{q^{2}+m_{3}^{2}} \cos \phi,-\sqrt{q^{2}+m_{3}^{2}} \sin \phi\right), \\
& e^{T}(3)=(0,-\sin \phi, \cos \phi),
\end{aligned}
$$




$$
\begin{aligned}
e^{L}(4) & =\frac{1}{m_{4}}\left(-q, \sqrt{q^{2}+m_{4}} \cos \phi, \sqrt{q^{2}+m_{4}^{2}} \sin \phi\right), \\
e^{T}(4) & =(0, \sin \phi,-\cos \phi) .
\end{aligned}
$$

\section{Appendix B. High-energy zero-norm states}

In this subsection, we explicitly calculate high-energy zero-norm states (HZNS) of some low-lying mass level. We will also show that the decoupling of these HZNS is equivalent to the decoupling of those spurious states used in the text to derive the desired linear relations. In the old covariant first quantization spectrum of open bosonic string theory, the solutions of physical state conditions include positive-norm propagating states and two types of zeronorm states. The latter are [7]

Type I: $\quad L_{-1}|x\rangle, \quad$ where $L_{1}|x\rangle=L_{2}|x\rangle=0, L_{0}|x\rangle=0$;

Type II: $\quad\left(L_{-2}+\frac{3}{2} L_{-1}^{2}\right)|\tilde{x}\rangle, \quad$ where $L_{1}|\tilde{x}\rangle=L_{2}|\tilde{x}\rangle=0,\left(L_{0}+1\right)|\tilde{x}\rangle=0$.

Based on a simplified calculation of higher mass level positive-norm states in [17], some general solutions of zero-norm states of Eqs. (B.1) and (B.2) at arbitrary mass level were calculated in [18]. Eqs. (B.1) and (B.2) can be derived from Kac determinant in conformal field theory. While type I states have zero-norm at any spacetime dimension, type II states have zero-norm only at $D=26$.

The solutions of Eqs. (B.1) and (B.2) up to the mass level $\mathrm{m}^{2}=4$ are listed as follows [18]:

(1) $m^{2}=-k^{2}=0$ :

$$
L_{-1}|x\rangle=k \cdot \alpha_{-1}|0, k\rangle, \quad|x\rangle=|0, k\rangle,|x\rangle=|0, k\rangle .
$$

(2) $m^{2}=-k^{2}=2$ :

$$
\begin{aligned}
& \left(L_{-2}+\frac{3}{2} L_{-1}^{2}\right)|\tilde{x}\rangle=\left[\frac{1}{2} \alpha_{-1} \cdot \alpha_{-1}+\frac{5}{2} k \cdot \alpha_{-2}+\frac{3}{2}\left(k \cdot \alpha_{-1}\right)^{2}\right]|0, k\rangle, \quad|\tilde{x}\rangle=|0, k\rangle, \\
& L_{-1}|x\rangle=\left[\theta \cdot \alpha_{-2}+\left(k \cdot \alpha_{-1}\right)\left(\theta \cdot \alpha_{-1}\right)\right]|0, k\rangle, \quad|x\rangle=\theta \cdot \alpha_{-1}|0, k\rangle, \theta \cdot k=0 .
\end{aligned}
$$

(3) $m^{2}=-k^{2}=4$ :

$$
\begin{aligned}
&\left(L_{-2}+\frac{3}{2} L_{-1}^{2}\right)|\tilde{x}\rangle=\left\{4 \theta \cdot \alpha_{-3}+\frac{1}{2}\left(\alpha_{-1} \cdot \alpha_{-1}\right)\left(\theta \cdot \alpha_{-1}\right)+\frac{5}{2}\left(k \cdot \alpha_{-2}\right)\left(\theta \cdot \alpha_{-1}\right)\right. \\
&\left.+\frac{3}{2}\left(k \cdot \alpha_{-1}\right)^{2}\left(\theta \cdot \alpha_{-1}\right)+3\left(k \cdot \alpha_{-1}\right)\left(\theta \cdot \alpha_{-2}\right)\right\}|0, k\rangle, \\
&|\tilde{x}\rangle=\theta \cdot \alpha_{-1}|0, k\rangle, k \cdot \theta=0,
\end{aligned}
$$




$$
\begin{aligned}
& L_{-1}|x\rangle= {\left[2 \theta_{\mu \nu} \alpha_{-1}^{\mu} \alpha_{-2}^{\nu}+k_{\lambda} \theta_{\mu \nu} \alpha_{-1}^{\lambda} \alpha_{-1}^{\mu} \alpha_{-1}^{\nu}\right]|0, k\rangle, } \\
&|x\rangle=\theta_{\mu \nu} \alpha_{-1}^{\mu \nu}|0, k\rangle, k \cdot \theta=\eta^{\mu \nu} \theta_{\mu \nu}=0, \theta_{\mu \nu}=\theta_{\nu \mu}, \\
& L_{-1}|x\rangle= {\left[\frac{1}{2}\left(k \cdot \alpha_{-1}\right)^{2}\left(\theta \cdot \alpha_{-1}\right)+2 \theta \cdot \alpha_{-3}+\frac{3}{2}\left(k \cdot \alpha_{-1}\right)\left(\theta \cdot \alpha_{-2}\right)\right.} \\
&\left.+\frac{1}{2}\left(k \cdot \alpha_{-2}\right)\left(\theta \cdot \alpha_{-1}\right)\right]|0, k\rangle, \\
&|x\rangle=[\left.2 \theta \cdot \alpha_{-2}+\left(k \cdot \alpha_{-1}\right)\left(\theta \cdot \alpha_{-1}\right)\right]|0, k\rangle, \theta \cdot k=0, \\
& L_{-1}|x\rangle= {\left[\frac{17}{4}\left(k \cdot \alpha_{-1}\right)^{3}+\frac{9}{2}\left(k \cdot \alpha_{-1}\right)\left(\alpha_{-1} \cdot \alpha_{-1}\right)+9\left(\alpha_{-1} \cdot \alpha_{-2}\right)\right.} \\
&\left.+21\left(k \cdot \alpha_{-1}\right)\left(k \cdot \alpha_{-2}\right)+25\left(k \cdot \alpha_{-3}\right)\right]|0, k\rangle, \\
&|x\rangle=\left[\frac{25}{2} k \cdot \alpha_{-2}+\frac{9}{2} \alpha_{-1} \cdot \alpha_{-1}+\frac{17}{4}\left(k \cdot \alpha_{-1}\right)^{2}\right]|0, k\rangle .
\end{aligned}
$$

Note that there are two degenerate vector zero-norm states, Eq. (B.6) for type II and Eq. (B.8) for type I, at mass level $m^{2}=4$. For mass level $m^{2}=2$, the high-energy limit of Eqs. (B.5) and (B.4) are calculated to be

$$
\begin{aligned}
& L_{-1}\left(\theta \cdot \alpha_{-1}\right)|0\rangle \rightarrow \sqrt{2} \alpha_{-1}^{L} \alpha_{-1}^{L}+\alpha_{-2}^{L}|0\rangle, \\
&\left(L_{-2}+\frac{3}{2} L_{-1}^{2}\right)|0\rangle \rightarrow\left(\sqrt{2} \alpha_{-2}^{L}+\frac{1}{2} \alpha_{-1}^{T} \alpha_{-1}^{T}\right)|0\rangle \\
&+\frac{3}{2}\left(2 \alpha_{-1}^{L} \alpha_{-1}^{L}+\sqrt{2} \alpha_{-2}^{L}\right)|0\rangle .
\end{aligned}
$$

Note that Eq. (B.12) is the high-energy limit of the second term of type II zero-norm state. It is easy to see that the decoupling of (B.10) implies the decoupling of (B.12). So one can neglect the effect of (B.12) even though it is of leading order in energy. It turns out that this phenomena persists to any higher mass level as well. This justifies that the decoupling of HZNS is equivalent to the decoupling of those spurious states used in the text to derive the desired linear relations. By solving Eqs. (B.10) and (B.11), we get the desired linear relation, $\mathcal{T}_{T T}: \mathcal{T}_{L}: \mathcal{T}_{L L}=4:-\sqrt{2}: 1$. Similarly, the high-energy limit of Eqs. (B.6)-(B.9) are calculated to be

$$
\begin{aligned}
& \left(L_{-2}+\frac{3}{2} L_{-1}^{2}\right)|0\rangle \rightarrow\left(4 \alpha_{-1}^{(T} \alpha_{-2}^{L)}+\frac{1}{2} \alpha_{-1}^{T} \alpha_{-1}^{T} \alpha_{-1}^{T}\right)|0\rangle \\
& +\frac{3}{2}\left(4 \alpha_{-1}^{(L} \alpha_{-1}^{L} \alpha_{-1}^{T)}+4 \alpha_{-1}^{(T} \alpha_{-2}^{L)}\right)|0\rangle, \\
& L_{-1}\left(\theta_{\mu \nu} \alpha_{-1}^{\mu \nu}\right)|0\rangle \rightarrow\left[2 \alpha_{-1}^{(T} \alpha_{-2}^{L)}+2 \alpha_{-1}^{(L} \alpha_{-1}^{L} \alpha_{-1}^{T)}\right]|0\rangle, \\
& L_{-1}\left[2 \theta \cdot \alpha_{-2}+\left(k \cdot \alpha_{-1}\right)\left(\theta \cdot \alpha_{-1}\right)\right]|0\rangle \rightarrow\left(4 \alpha_{-1}^{(L} \alpha_{-1}^{L} \alpha_{-1}^{T)}+4 \alpha_{-1}^{(T} \alpha_{-2}^{L)}\right)|0\rangle, \\
& L_{-1}\left[\frac{25}{2} k \cdot \alpha_{-2}+\frac{9}{2} \alpha_{-1} \cdot \alpha_{-1}+\frac{17}{4}\left(k \cdot \alpha_{-1}\right)^{2}\right]|0\rangle \rightarrow 0 .
\end{aligned}
$$


It is easy to see that the decoupling of Eq. (B.15) or (B.16) implies the decoupling of Eq. (B.14). By solving the equations, one gets $\mathcal{T}_{T T T}: \mathcal{T}_{L L T}: \mathcal{T}_{(L T)}: \mathcal{T}_{[L T]}=8: 1:-1: 1$.

\section{Appendix C. Virasoro constraints}

\section{C.1. High-energy limit of Virasoro constraints}

To take the high-energy limit for the Virasoro constraints, we replace the indices $\left(\mu_{i}, v_{i}\right)$ by $L$ or $T$, and

$$
k^{\mu_{i}} \rightarrow \hat{m} e^{L}, \quad \eta^{\mu_{1} \mu_{2}} \rightarrow e^{T} e^{T} .
$$

Eqs. (63a) and (63b) become

$$
\begin{aligned}
& 0=\hat{m} \begin{array}{|l|l|l|l|}
\hline L & \mu_{2}^{1} & \cdots & \mu_{m_{1}}^{1}
\end{array} \bigotimes_{j \neq 1}^{k} \begin{array}{|l|l|l|}
\hline \mu_{1}^{j} & \cdots & \mu_{m_{j}}^{j} \\
\hline
\end{array} \\
& +\sum_{i=2}^{m_{1}} \begin{array}{|l|l|l|l|l|}
\hline \mu_{2}^{1} & \cdots & \hat{\mu}_{i}^{1} & \cdots & \mu_{m_{1}}^{1}
\end{array} \otimes \begin{array}{|l|l|l|l|}
\hline \mu_{i}^{1} & \mu_{1}^{2} & \cdots & \mu_{m_{2}}^{2}
\end{array} \bigotimes_{j \neq 1,2}^{k} \begin{array}{|l|l|l|}
\mu_{1}^{j} & \cdots & \mu_{m_{j}}^{j} \\
\hline
\end{array} \\
& +\sum_{l=3}^{k}(l-1) \begin{array}{|l|l|l|}
\mu_{2}^{1} & \cdots & \mu_{m_{1}}^{1}
\end{array} \otimes \sum_{i=1}^{m_{l-1}} \begin{array}{|l|l|l|l|l|}
\mu_{1}^{l-1} & \cdots & \hat{\mu}_{i}^{l-1} & \cdots & \mu_{m_{l-1}}^{l-1} \\
\hline
\end{array} \\
& \otimes \begin{array}{|l|l|l|l|}
\hline \mu_{i}^{l-1} & \mu_{1}^{l} & \cdots & \mu_{m_{l}}^{l} \\
\hline
\end{array} \bigotimes_{j \neq 1, l, l-1}^{k} \begin{array}{|l|l|l|}
\mu_{1}^{j} & \cdots & \mu_{m_{j}}^{j} \\
\hline
\end{array}
\end{aligned}
$$

and

$$
\begin{aligned}
& 0=\frac{1}{2} \begin{array}{|l|l|l|l|l|}
\hline T & T & \mu_{3}^{1} & \cdots & \mu_{m_{1}}^{1}
\end{array} \bigotimes_{j \neq 1}^{k} \begin{array}{|l|l|l|}
\mu_{1}^{j} & \cdots & \mu_{m_{j}}^{j} \\
\hline
\end{array} \\
& +\hat{m} \begin{array}{|l|l|l|}
\hline \mu_{3}^{1} & \cdots & \mu_{m_{1}}^{1}
\end{array} \otimes \begin{array}{|l|l|l|l|}
\hline \mu_{1}^{2} & \cdots & \mu_{m_{2}}^{2} & L \\
\hline
\end{array} \bigotimes_{j \neq 1,2}^{k} \begin{array}{|l|l|l|}
\mu_{1}^{j} & \cdots & \mu_{m_{j}}^{j} \\
\hline
\end{array} \\
& +\sum_{i=3}^{m_{1}} \begin{array}{|l|l|l|l|l|}
\hline \mu_{3}^{1} & \cdots & \hat{\mu}_{i}^{1} & \cdots & \mu_{m_{1}}^{1}
\end{array} \otimes \begin{array}{|l|l|l|l|l|l|l|l|}
\hline \mu_{i}^{1} & \mu_{1}^{3} & \cdots & \mu_{m_{3}}^{3} & \bigotimes & \bigotimes_{j \neq 1,3}^{k} & \cdots & \mu_{m_{j}}^{j} \\
\hline
\end{array} \\
& +\sum_{l=4}^{k}(l-2) \begin{array}{|l|l|l|l|}
\mu_{3}^{1} & \cdots & \mu_{m_{1}}^{1}
\end{array} \otimes \sum_{i=1}^{m_{l-2}} \begin{array}{|l|l|l|l|l|}
\mu_{1}^{l-2} & \cdots & \hat{\mu}_{i}^{l-2} & \cdots & \mu_{m_{l}}^{l-2} \\
\hline
\end{array} \\
& \otimes \begin{array}{|l|l|l|l|}
\hline \mu_{i}^{l-2} & \mu_{1}^{l} & \cdots & \mu_{m_{l}}^{l} \\
\hline
\end{array} \bigotimes_{j \neq 1, l, l-2}^{k} \begin{array}{|l|l|l|}
\mu_{1}^{j} & \cdots & \mu_{m_{j}}^{j} \\
\hline
\end{array}
\end{aligned}
$$


The indices and $\left\{\mu_{i}^{j}\right\}$ are symmetric and can be chosen to have $l_{j}$ of $\{L\}$ which $0 \leqslant l_{j} \leqslant m_{j}$ and $\{T\}$ for the rest. Thus

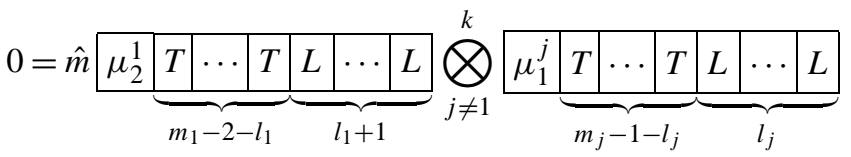

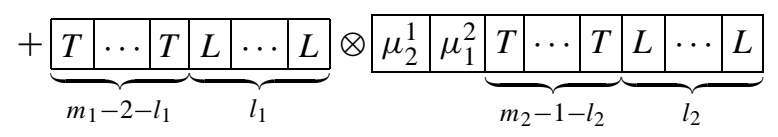

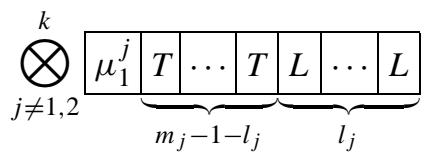

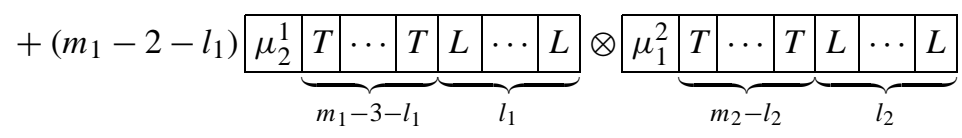

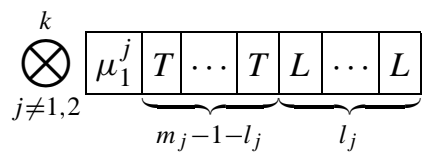

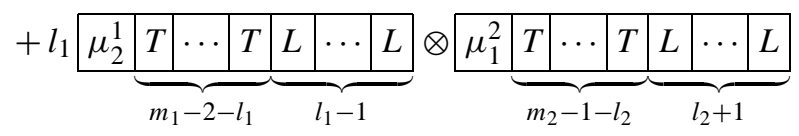

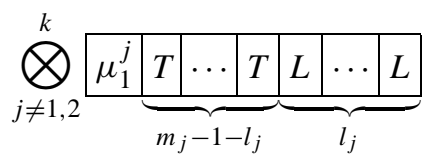

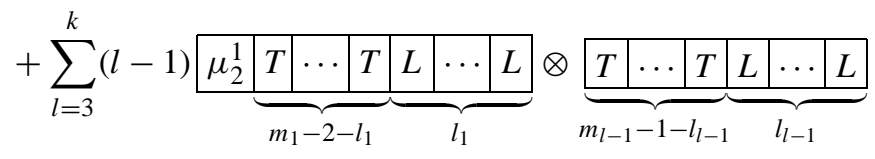

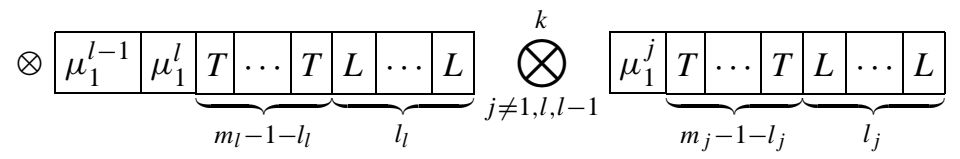

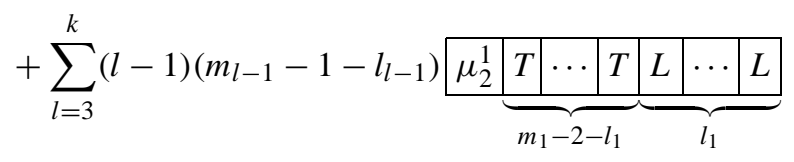

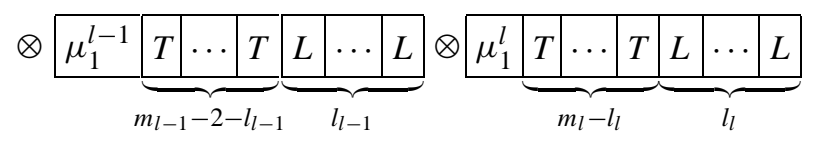




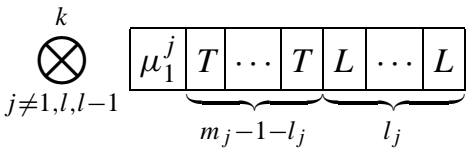

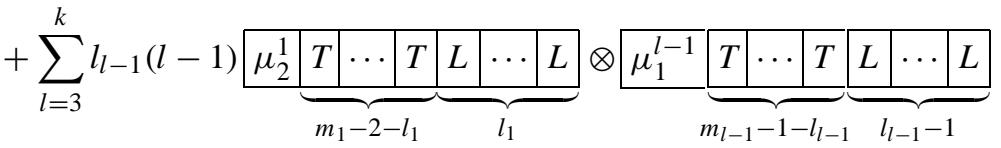

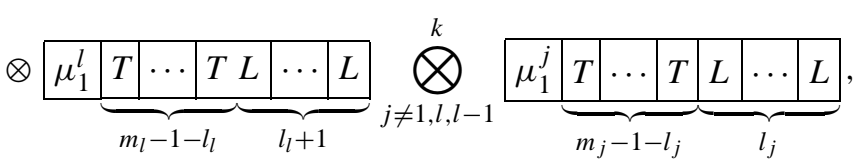

and

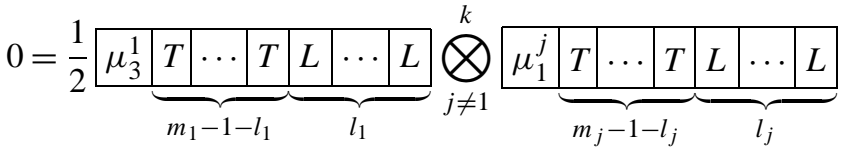

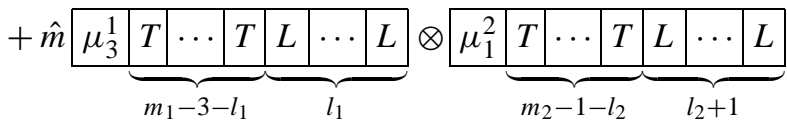

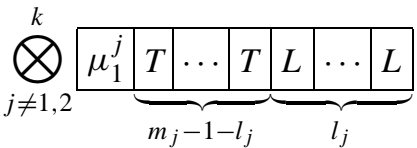

$$
\begin{aligned}
& +\begin{array}{l|l|l|l|l|l|}
\hline T & \ldots & T & L & \cdots & L
\end{array} \otimes \begin{array}{ll|l|l|l|l|l|l|l|}
\hline \mu_{3}^{1} & \mu_{1}^{3} & T & \cdots & T & L & \cdots & L \\
\hline m_{1}-3-l_{1} & \underbrace{}_{l_{1}}
\end{array}
\end{aligned}
$$

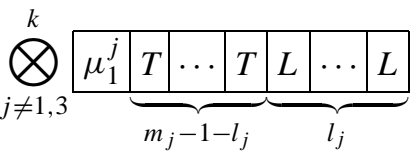

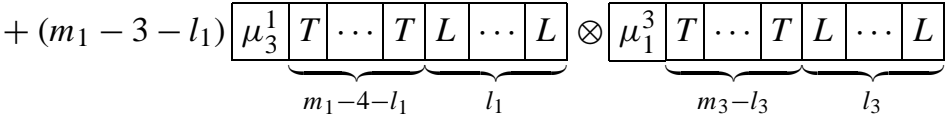

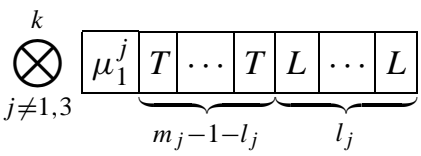

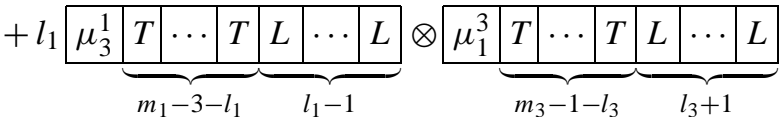

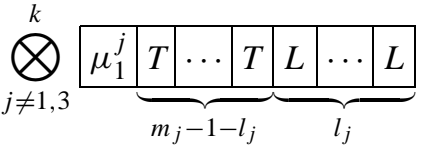




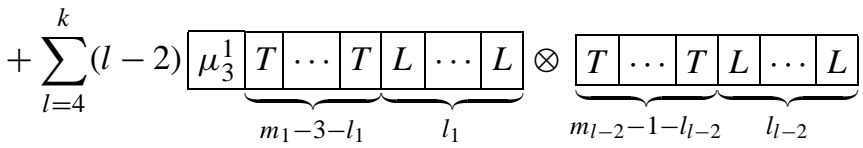

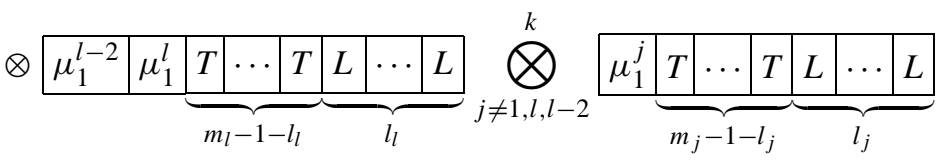

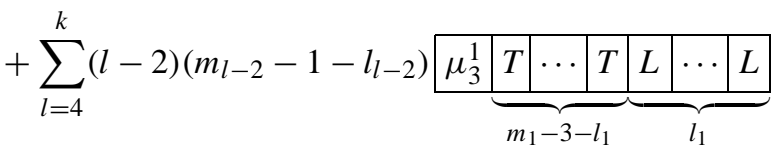

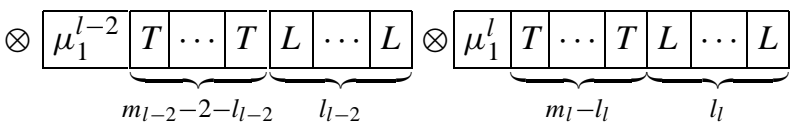

$$
\begin{aligned}
& \bigotimes_{j \neq 1, l, l-2}^{k} \begin{array}{|l|l|l|l|l|l|l|}
\mu_{1}^{j} & T & \cdots & T & L & \cdots & L \\
m_{m_{j}}-1-l_{j} & \underbrace{}_{l_{j}}
\end{array}
\end{aligned}
$$

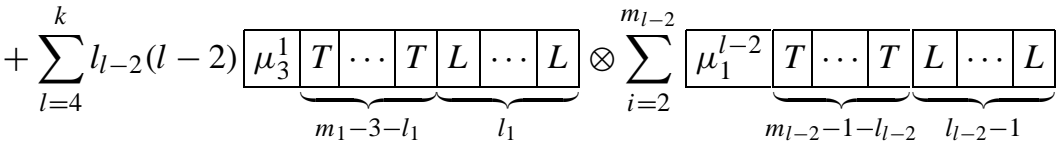

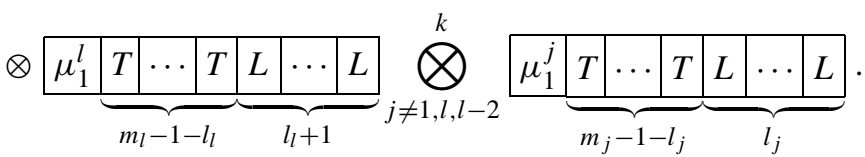

There are still some undetermined parameters $\mu_{2}^{1}, \mu_{3}^{1}$ and $\mu_{1}^{j}(j \geqslant 2)$, which can be chosen to be $L$ or $T$, in the above equations. However, it is easy to see that both choices lead to the same equations. Therefore, we will set all of them to be $T$ in the following. The final Virasoro constraints at high energy become

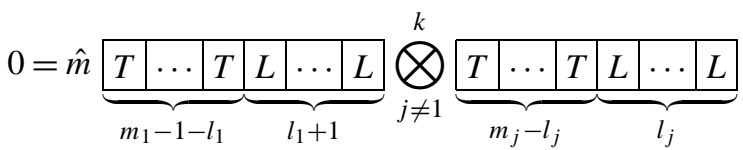

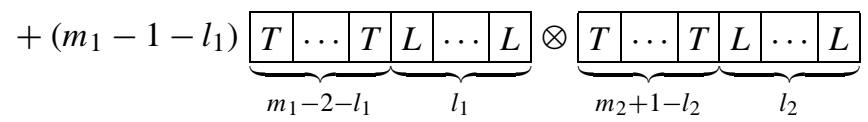

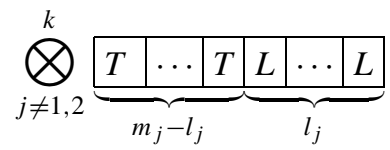

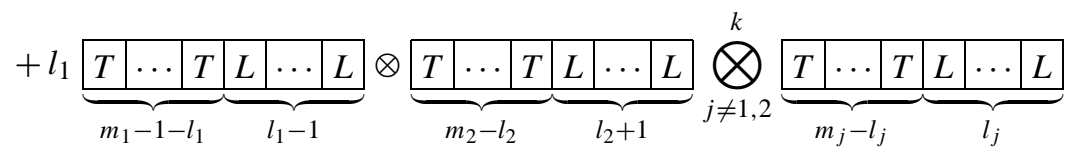




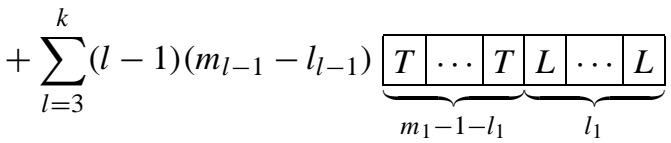

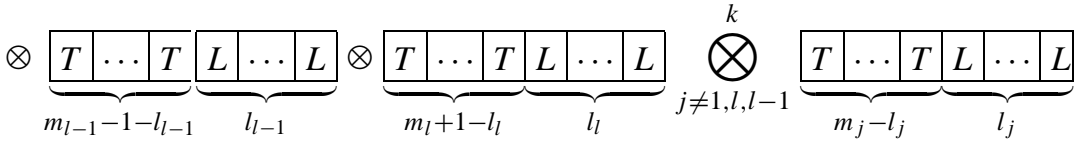

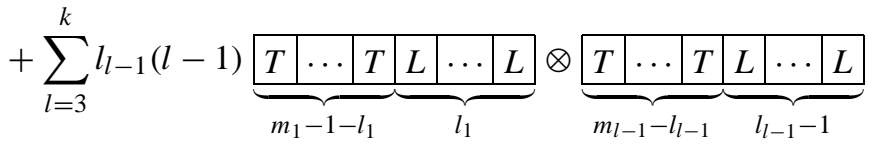

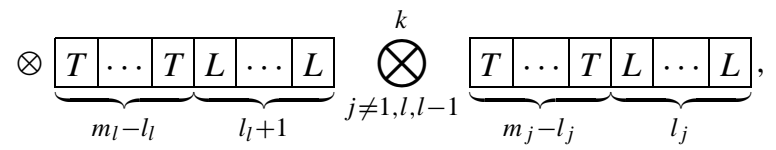

and

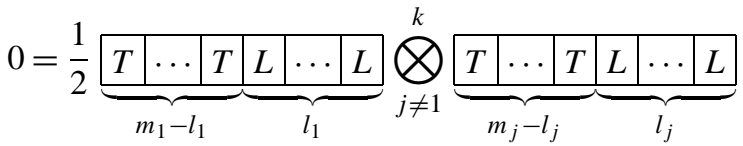

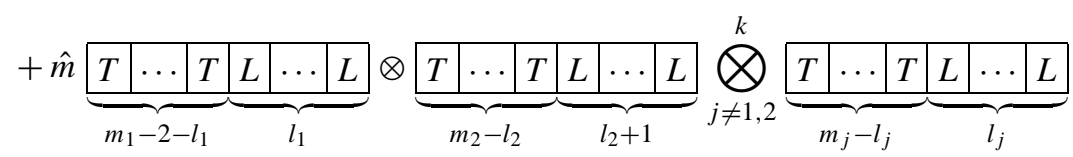

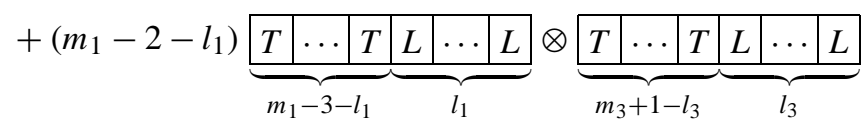

$$
\begin{aligned}
& \bigotimes_{j \neq 1,3}^{k} \underbrace{\begin{array}{l|l|l|l|l|l|}
T & \cdots & T & L & \cdots & L \\
l_{j}
\end{array}}_{m_{j}-l_{j}}
\end{aligned}
$$

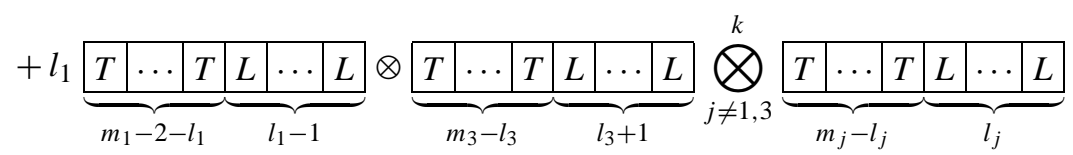

$$
\begin{aligned}
& +\sum_{l=4}^{k}(l-2)\left(m_{l-2}-l_{l-2}\right) \underbrace{\begin{array}{ll|l|l|l|l|}
T & \cdots & T & L & \cdots & L \\
l_{1}
\end{array}}_{m_{1}-2-l_{1}}
\end{aligned}
$$

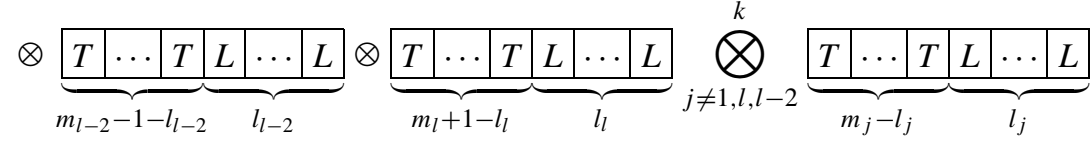

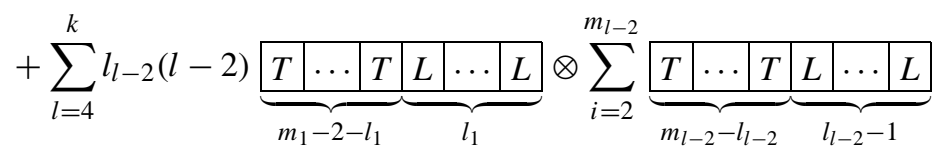




$$
\otimes \underbrace{\begin{array}{|l|l|l|l|l|l|}
T & \cdots & T & L & \cdots & L \\
l_{l}+1
\end{array}}_{m_{l}-l_{l}} \bigotimes_{j \neq 1, l, l-2}^{k} \underbrace{\begin{array}{ll|l|l|l|l|l|}
T & \cdots & T & L & \cdots & L \\
l_{j}
\end{array}}_{m_{j}-l_{j}} .
$$

\section{C.2. Proof of the lemma (66)}

In this subsection, we prove the lemma given in Section 4.2 as follows

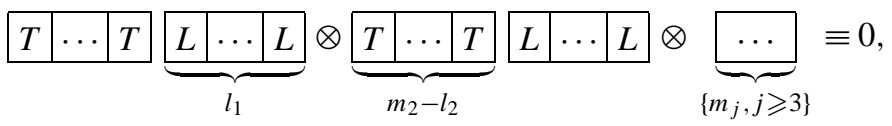

except for (i) $l_{2}=m_{2}, m_{j}=0$ for $j \geqslant 3$ and (ii) $l_{1}=2 m$.

Proof. In the high-energy limit, we only need to consider the leading energy terms. To count the energy scaling behavior, the rule is the same as Eq. (20): each $T$ contributes a factor of energy $E$ and each $L$ contributes $E^{2}$. Any terms with total energy order level less than $n$ are sub-leading terms and can be ignored.

(i) If $l_{2} \neq m_{2}$ and $m_{j} \neq 0, j \geqslant 3$, then in Eq. (C.4a),

(1) for $l_{1}=0$, all terms except the first term are sub-leading, then

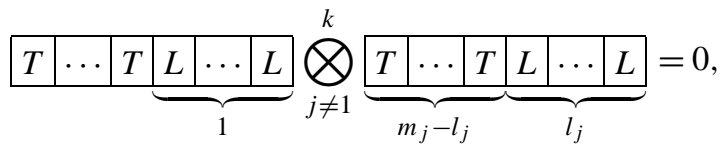

(2) for $l_{1}=1$, the third term is sub-leading, and (C.6) implies all other terms except the first term are vanished, then

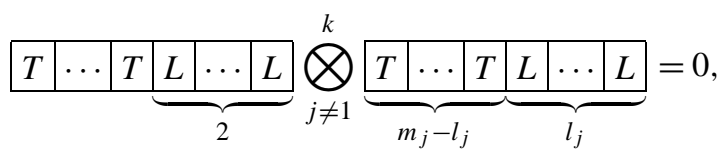

(3) if for $l_{1}=l^{\prime}$,

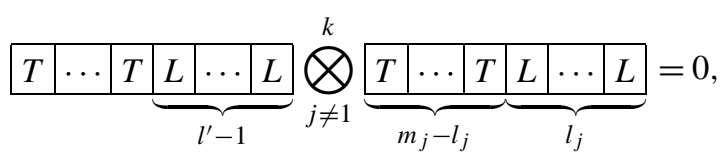

and

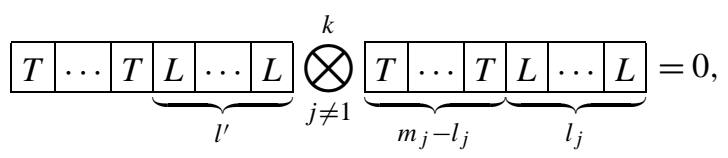

(C.4a) implies all terms except the first term are vanished, then

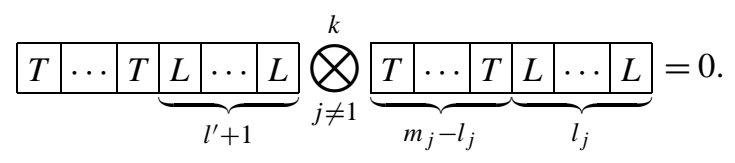


(ii) If $l_{2}=m_{2}$ and $m_{j}=0$ for $j \geqslant 3$, then Eq. (C.4a) reduces to

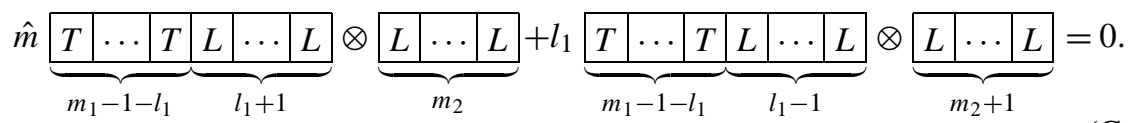

Similarly, we have in Eq. (C.11),

(1) for $l_{1}=0$,

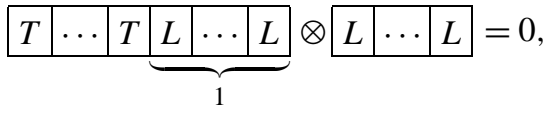

(2) if for $l_{1}=2 m$,

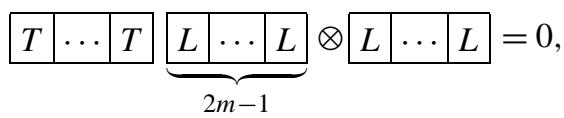

then (C.11) implies

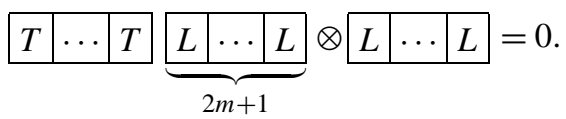

\section{References}

[1] D.J. Gross, P. Mende, Phys. Lett. B 197 (1987) 129;

D.J. Gross, P. Mende, Nucl. Phys. B 303 (1988) 407.

[2] D.J. Gross, High energy symmetry of string theory, Phys. Rev. Lett. 60 (1988) 1229;

D.J. Gross, Philos. Trans. R. Soc. London A 329 (1989) 401.

[3] D.J. Gross, J.L. Manes, The high energy behavior of open string theory, Nucl. Phys. B 326 (1989) 73, see Section 6 for details.

[4] J.C. Lee, Phys. Lett. B 326 (1994) 79;

M. Evans, B.A. Ovrut, Phys. Lett. B 231 (1989) 80.

[5] See, for instance, J. Isberg, U. Lindstrom, B. Sundborg, G. Theodoridis, Classical and quantized tensionless strings, Nucl. Phys. B 411 (1994) 122, hep-th/9307108;

B. Sundborg, Stringy gravity, interacting tensionless strings and massless higher spins, Nucl. Phys. B (Proc. Suppl.) 102 (2001) 113, hep-th/0103247;

E. Sezgin, P. Sundell, Massless higher spins and holography, Nucl. Phys. B 644 (2002) 303, hep-th/0205131; E. Sezgin, P. Sundell, Nucl. Phys. B 660 (2003) 403, Erratum;

C.S. Chu, P.M. Ho, F.L. Lin, Cubic string field theory in pp-wave background and background independent Moyal structure, JHEP 0209 (2002) 003, hep-th/0205218;

G. Bonelli, On the tensionless limit of bosonic strings, infinite symmetries and higher spins, Nucl. Phys. B 669 (2003) 159, hep-th/0305155.

[6] See, for instance, C. Fronsdal, Massless fields with integer spin, Phys. Rev. D 18 (1978) 3624;

J. Fang, C. Fronsdal, Massless fields with half integral spin, Phys. Rev. D 18 (1978) 3630;

M.A. Vasiliev, Progress in higher spin gauge theories, Prepared for 9th Marcel Grossmann Meeting on Recent Developments in Theoretical and Experimental General Relativity, Gravitation and Relativistic Field Theories (MG 9), Rome, Italy, 2-9 July 2000;

M.A. Vasiliev, Higher spin gauge theories in various dimensions, Fortschr. Phys. 52 (2004) 702, hepth/0401177. 
[7] B. Green, J.H. Schwarz, E. Witten, Superstring Theory, Cambridge Monographs on Mathematical Physics, vol. I, Cambridge Univ. Press, Cambridge, 1988 (Chapter 2).

[8] H.C. Kao, J.C. Lee, Phys. Rev. D 67 (2003) 086003.

[9] C.T. Chan, J.C. Lee, Y. Yang, Anatomy of zero-norm states in string theory, Phys. Rev. D 71 (2005) 086005, hep-th/0501020.

[10] J.C. Lee, Phys. Lett. B 241 (1990) 336;

J.C. Lee, Phys. Rev. Lett. 64 (1990) 1636;

J.C. Lee, B. Ovrut, Nucl. Phys. B 336 (1990) 222.

[11] J.C. Lee, Prog. Theor. Phys. 91 (1994) 353;

J.C. Lee, Phys. Lett. B 337 (1994) 69.

[12] C.T. Chan, J.C. Lee, Stringy symmetries and their high-energy limits, Phys. Lett. B 611 (2005) 193, hepth/0312226.

[13] C.T. Chan, J.C. Lee, Nucl. Phys. B 690 (2004) 3, hep-th/0401133.

[14] C.T. Chan, P.M. Ho, J.C. Lee, Ward identities and high-energy scattering amplitudes in string theory, Nucl. Phys. B 708 (2005) 99, hep-th/0410194.

[15] T.D. Chung, J.C. Lee, Discrete gauge states and $w_{\infty}$ charges in $c=12$ D gravity, Phys. Lett. B 350 (1995) 22, hep-th/9412095;

T.D. Chung, J.C. Lee, Superfield form of discrete gauge states in $\hat{c}=12$ d supergravity, Z. Phys. C 75 (1997) 555, hep-th/9505107;

J.C. Lee, Eur. Phys. J. C 1 (1998) 739.

[16] J. Avan, A. Jevicki, Phys. Lett. B 266 (1991) 35;

J. Avan, A. Jevicki, Phys. Lett. B 272 (1991) 17;

I.R. Klebanov, A.M. Polyakov, Mod. Phys. Lett. A 6 (1991) 3273.

[17] J.L. Manes, M.A.H. Vozmediano, Nucl. Phys. B 326 (1989) 271.

[18] J.C. Lee, Calculations of zero-norm states and reduction of stringy scattering amplitudes, hep-th/0302123. 